\title{
THE EVOLUTION OF GENETIC DISCONTINUITY: COMPUTER MODELS OF THE SELECTION OF BARRIERS TO INTERBREEDING BETWEEN SUBSPECIES
}

\author{
JACK L. CROSBY \\ Department of Botany, University of Durham
}

Received 30.vi.69

\section{Introduction}

\begin{abstract}
"The individuals of a genus spread out over continents and become widely separated. Through differences in location, food and soil, they form varieties which because of their isolation never cross with other varieties, and so cannot revert in that way to the original type. Finally, they achieve constancy and become new species. Then perhaps by some other route they reach the new territory of a variety which has been similarly altered; both are now very different species which will not again interbreed with one another."
\end{abstract}

WRITTEN in 1825 by Leopold von Buch, this account of the process of allopatric speciation would be acceptable to many biologists today. This paper deals with one aspect of allopatric speciation.

Broadly speaking, when two geographical subspecies (of the same species, which will always be implied throughout this paper) come into contact with one another, one of four situations may arise.

They may freely interbreed, producing hybrids with little loss of viability or fertility. This would lead to free gene flow between the subspecies which would lose their separate identity, with the re-establishment of a single gene pool.

They may interbreed, producing hybrids with reduced viability or fertility. This is the situation which is to be examined theoretically in this paper.

They may interbreed with the production of sterile hybrids. There would be no gene flow; subspecific identity and genetic discontinuity would be maintained. The subspecies would then be regarded as distinct species.

Finally, the situation described by von Buch, they might not interbreed at all.

The situation in which hybridisation occurs, with at least some fertility of the hybrids, has been discussed by Dobzhansky (1940), and subsequently by a number of other people. Dobzhansky considered that such hybridisation would jeopardise the " integrity" of geographical races or subspecies, and suggested that genetic factors which would decrease or prevent interbreeding would have a selective advantage because they would help to preserve such integrity. But integrity, the maintenance of an uncontaminated racial or subspecific genotype, has no intrinsic value. The consequences of loss of integrity may be good or bad according to circumstances, and it is the consequences we have to consider, not some hypothetical idealistic quality. If the subspecies meet in an environment which is not optimal for either of them, hybridisation may mean loss of integrity but it could allow the pooling of genetic resources and the selection of a new genotype better adapted to that environment than either of the pure subspecies. 
On the other hand, where interbreeding leads to the formation of hybrids with reduced fertility, then this involves wastage of gametes which participate in hybrid formation. It is this gametic wastage and not the loss of integrity which is disadvantageous and which may be expected to confer a selective advantage on genes which restrict or prevent interbreeding. As Mayr (1963) has pointed out, such selective advantage should be greatest where the hybrids are completely sterile; that is, when there could be no gene flow between the species and no danger of loss of integrity.

Speciation involves the formation of genetic barriers to gene flow between the incipient species. Hybrid sterility is a very effective barrier, but while it is certainly powerful in the maintenance of genetic integrity, it is difficult to see how it could come about as a result of selection. Any genotype inducing hybrid sterility (or any other post-fertilisation barrier to intersubspecific gene flow) is lost with the hybrid which it renders sterile, and is therefore selected against. Increase in hybrid sterility leads to an increase in gamete wastage and in that respect is disadvantageous rather than advantageous.

There might be an exception to this in a situation where two subspecies are competing for territory. If one does not hybridise as female, but does so as male with the other as female, then if those hybrids have low fertility there will be a reduction in numbers of the second subspecies and the first may benefit from this aggressive hybridisation (Crosby, 1966a). The problem merits deeper consideration, but within the broad context of allopatric speciation it would appear that genetic situations which can lead to hybrid sterility arise in subspecies while they are not in contact and from genetic changes which have no selective relevance to hybridisation.

Pre-fertilisation barriers to gene flow are quite different. By preventing hybridisation they avoid gamete wastage from that cause, and since the organisms carrying the desired genotypes are now those which reproduce most effectively, those genotypes can be selected instead of being lost in sterile hybrids. Pre-fertilisation isolating barriers occur more commonly in animals than in plants; accurate species recognition is an important and well-known example in animals, but can hardly occur directly in plants though indirectly it might perhaps be found in plants pollinated by insects with good powers of taxonomic discrimination.

Clearly, the idea that complete or partial sterility of inter-subspecific hybrids could lead to selection of genes tending to prevent interbreeding and the formation of hybrids, and consequently to acceleration of speciation and the establishment of genetic discontinuity, is a plausible one although it has not found favour with everybody. The arguments have been discussed at length by Mayr (1963) and will not be considered further in this paper, which will concentrate on the purely theoretical question of whether such selection is possible, and if so whether it can be effective.

The selection of genes whose advantage lies in the prevention of gamete wastage through hybridisation is a second-order effect, in the sense that the genes themselves do not confer any direct advantage or disadvantage, but allow the avoidance of a situation which has a consequential disadvantage. Of itself, hybridisation may not matter; it is the subsequent tendency to sterility of the hybrids which matters.

Second-order selection is a notoriously deceptive process when considered superficially, since its selective advantages are of a much lower order 
than those commonly found in the more familiar situations involving direct selection. Fisher's theory of the evolution of dominance is a case in point (Crosby, 1963).

The problem would seem to be beyond the reach of theoretical treatment by the orthodox methods of mathematics, which can only cope adequately with evolutionary systems which are, by biological standards, relatively simple. Even if mathematical treatment of this problem were possible, it would certainly require a level of mathematical complexity beyond the comprehension of almost all biologists, who would be in no position to assess either the biological sense or the accuracy of the mathematical interpretation.

By contrast, computer simulation (Grosby, 1961, 1967) provides an excellent technique for the theoretical investigation of quite complex evolutionary systems, entirely avoiding mathematics and the consequent difficulties of assessing the validity or otherwise of algebraic and statistical representations of the evolutionary processes involved. "Words" of a computer may be used as coded representations of the genotypes of organisms, and thus as models of the organisms themselves. Model populations may be composed of arrays of such model organisms, which have real individuality and which grow, interbreed, reproduce and die according, for example, to such laws of genetics, incidences of selection and chance, and rules about breeding behaviour as we may choose, in a way which is strictly analogous to the behaviour of real organisms in real populations. Assumptions made in constructing such a model system may easily be stated and examined for their biological validity; there are no mathematical assumptions or interpretations to be considered. The stochastic element, which is an essential ingredient of all biological systems, is easily incorporated by the proper use of pseudo-random number sequences. Interpretation of the results from such a model does require a clear idea of the biological principles involved, and an imaginative ability to regard the populations as composed of individuals which, even if electronic, are none the less real.

The preliminary experiments which are about to be described were carried out on a Ferranti Pegasus computer. Subsequently, a larger and very much faster English Electric KDF9 became available, and this was used for the more complex experiments described in section 3 onwards.

\section{The SELEction OF AN ISOLATING SYSTEM}

In planning the series of experiments which are now to be described, I decided that their basis should be as realistic as possible. Rather than consider some wholly hypothetical organism, I chose to deal with flowering plants as the group of organisms with whose biology I was most familiar.

The preliminary experiments imagined a situation in which, following a period of geographical isolation and genetic divergence, a species had come to consist of two subspecies (which will be referred to as $A$ and $B$ ), genetically differentiated to such an extent that while they could freely intercross if given the opportunity, the hybrids so formed had a considerably impaired fertility. A and B had come together and overlapped, and the computer model simulated a small population in which they were equally numerous (256 of each) and thoroughly intermingled at random; dispersal of pollen and seed was random through the population.

Flowering time was chosen to be the potential pre-fertilisation barrier to 
interbreeding. This is quite a common isolating barrier; for example, Primula vulgaris and $P$. veris, which often occur together in Britain, hybridise less frequently in the South of England, where the two species have a relatively small overlap in their flowering period, than in the North, where $P$. vulgaris is much more delayed in flowering than $P$. veris and there is a much bigger overlap in period of flowering.

There are two ways in which a change of flowering period may come about. One is by change in the mean date of flowering; the other by shortening of the period of flowering. If two hybridising subspecies, flowering at similar times, are going to develop a divergence in their flowering times, we would expect one to bring forward flowering and the other to delay it, and both to shorten their period of flowering provided this does not involve any significant loss of reproductive capacity.

If there is already some difference in flowering time between two subspecies which hybridise freely and show poor hybrid fertility, it is easy to see how natural selection could amplify this difference.

We will consider gamete wastage in terms of ovules only. Suppose that A is tending to flower slightly earlier than B, although there is still substantial overlap. Earlier in the season there will be relatively more A pollen than B; later, the proportion of B pollen will increase, and at the end there will be relatively more $B$ pollen than $A$. At the beginning of the season, most ovules of $\mathrm{A}$ plants will be fertilised by $\mathrm{A}$ pollen, but the later an $\mathrm{A}$ plant flowers the higher will be the proportion of its ovules which will be fertilised by B pollen and so subject to the wastage of hybridisation. There should be a selective advantage to $A$ in early flowering and, by a corresponding argument, to $B$ in late flowering.

Further, provided there is no consequential loss of reproductive capacity, it should be advantageous for both $A$ and $B$ to shorten the flowering period, since this would reduce the period of overlap.

While there remains substantial overlap in the flowering periods of the two subspecies, the greater the difference in mean flowering time between them the greater the advantage of earliness to $A$ and lateness to $B$. We would expect a positive feedback situation, with evolutionary progress slow at first but accelerating with increasing divergence.

On the other hand, if the flowering periods of A and B were initially identical, there could be no selection pressure which would induce a divergence between $A$ and $B$, and no such pressure could exist until such a divergence arises. Although divergence could not be initiated by selection, the situation is a metastable one and the stability could be upset by random fluctuation if the genetic potential for variation were there. As soon as fluctuation-induced divergence exists, then selection pressure should come into action.

In these early experiments it was decided that the two subspecies should initially flower simultaneously, to test the supposition that random fluctuation could produce a deviation in flowering time sufficient to initiate a selective process.

The plants were treated as annuals with no seed dormancy, all those flowering in any one year having grown from seed produced in the previous year. There was thus no overlapping of generations. The plants were imagined to be entomophilous hermaphrodites breeding at random, and the only possible barrier to cross-fertilisation between the two subspecies 
was flowering time; that is, the pollinating insects were totally lacking in taxonomic discrimination at the subspecific level.

\section{(i) The genetics of the situation}

\section{(a) Hybrid fertility}

The two subspecies $\mathrm{A}$ and $\mathrm{B}$ were characterised by 8 independent loci, collectively referred to as the $G$ loci, A being $G_{1} G_{1} G_{2} G_{2} \ldots G_{8} G_{8}$ and B being $g_{1} g_{1} g_{2} g_{2} \ldots g_{8} g_{8}$. The only phenotypic effect of these genes was on fertility. There was no dominance, all $G$ alleles were identical in effect, all $g$ alleles were identical in effect, and the effects were strictly additive. For example,

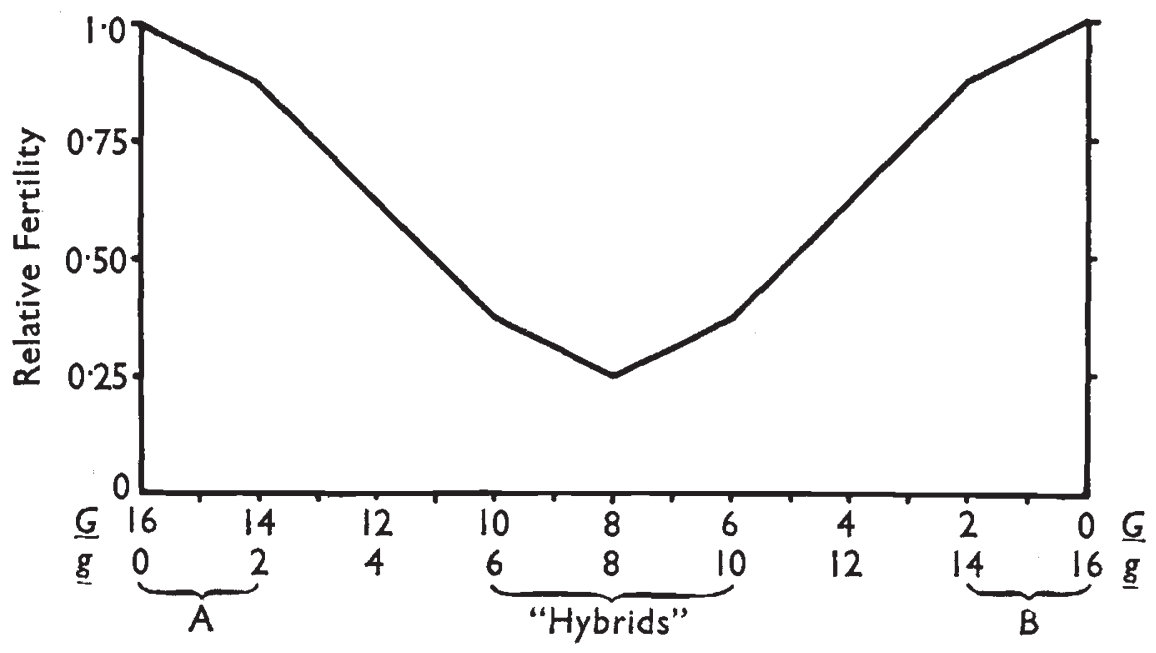

FIG. 1.-Diagram showing the relative fertilities of the various genotypes, as used in the pilot experiment and in run 16 (see section 3 ). The genotypes corresponding to A, B and hybrids, as these terms are generally used in the text, are indicated at the base of the diagram.

$G_{1} G_{1} G_{2} g_{2} G_{3} g_{3} G_{4} G_{4} G_{5} g_{5} g_{6} g_{6} G_{7} g_{7} G_{8} G_{8}$ and $G_{1} g_{1} G_{2} g_{2} G_{3} G_{3} G_{4} G_{4} g_{5} g_{5} G_{6} g_{6}$ $G_{7} G_{7} G_{8} g_{8}$ would be identical and could be expressed phenotypically as $10 G 6 g$. Pure A can be referred to as $16 G$, pure $\mathrm{B}$ as $16 g$ and the $\mathrm{F}_{1}$ hybrid as $8 G 8 g$. A and $\mathrm{B}$, without qualification, may usually be taken to include plants as impure as $14 G 2 g$ and $2 G 14 g$ respectively.

The $\mathrm{F}_{1}$ of course is heterozygous at all 8 loci, but other $8 \mathrm{G} 8 \mathrm{~g}$ individuals could occur, for example in an $F_{2}$ family. Whatever the precise genotype, all $8 G 8 g$ individuals had the same fertility, which was $0 \cdot 25$, that of each pure subspecies being 1.0 . Individuals intermediate between the pure subspecies and $8 G 8 \mathrm{~g}$ had intermediate fertilities, as shown in fig. 1 .

Fertility was the only criterion for selection. It was assumed that all seeds were equally viable.

\section{(b) Time of flowering}

The flowering period genes were divided into two groups.

Three independent loci, referred to as the $D$ loci, were concerned with the time of commencement of flowering. There was no dominance, all $D$ alleles were identical in effect, as were all $d$ alleles, and the effects were R 2 
strictly additive. There were eight consecutive weeks during which flowering was possible. $D$ alleles promoted early flowering; $6 D$ plants would begin to flower in the first week; $6 d$ plants would finish flowering in the last week.

Two independent loci, the $W$ loci, determined the number of consecutive weeks during which a plant was flowering. Again there was no dominance, all $W$ alleles were identical in effect, as were all $w$ alleles, and the effects were strictly additive. The minimum length of flowering was two weeks, determined by a genotype of $4 W ; 4 w$ plants flowered for the maximum time of six weeks.

The simplifying assumption was made that any plant would flower for an exact number of weeks, that all plants coming into flower in any particular week would start simultaneously at the beginning of that week, and that all plants finishing their flowering in a particular week would do so simultaneously at the end of that week. The total number of flowers produced by a plant was independent of the number of weeks during which it flowered, and any individual plant produced the same number of flowers during each of its weeks of flowering. Thus a $4 W$ plant would produce three times as many flowers in a week as a $4 w$ plant of equal fertility.

There was no linkage, and no interaction, between any genes in the three groups $G, D$ and $W$.

\section{(ii) Procedure for dealing with fertility}

Each plant was assigned a weekly fertility value (maximum $1 \cdot 0$ ) based on its actual fertility and the number of weeks of flowering. For any plant, the fertilities of pollen and ovules were identical. Fertility can be thought of

TABLE 1

Examples showing how the value for weekly fertility was derived

$\begin{array}{lccc}\text { Genotype } & \begin{array}{c}\text { Actual } \\ \text { fertility }\end{array} & \begin{array}{c}\text { Weeks of } \\ \text { flowering }\end{array} & \begin{array}{c}\text { Weekly } \\ \text { fertility }\end{array} \\ 16 G 4 W & 1.0 & 2 & 1.0 \\ 16 G 2 W 2 w & 1.0 & 4 & 1.0 \times 2 / 4=0.5 \\ 16 G 4 w & 1.0 & 6 & 1.0 \times 2 / 6=0.33 \\ 13 G 3 g 3 W 1 w & 0.75 & 3 & 0.75 \times 2 / 3=0.5 \\ 8 G 8 g 4 W & 0.25 & 2 & 0.25 \\ 8 G 8 g 1 W 3 w & 0.25 & 5 & 0.25 \times 2 / 5=0.1\end{array}$

in terms of the goodness of pollen and ovules, with total flower production being equal for all genotypes, that is, the reproductive capacity of a flower would be equivalent to the fertility of the plant. An alternative way of considering fertility would be to consider that flowers of all genotypes had equal reproductive capacity but that the total number of flowers produced by a plant corresponded to its fertility. There is no essential difference between these two views; the former is a more likely interpretation, but it may be simpler to think of the model in terms of the latter.

The examples given in table 1 illustrate the way in which the weekly fertility was arrived at.

Reproduction was carried out on a week-by-week basis. The number of seeds to be produced in any week was a fraction of the total seeds (512) required to reproduce the population, this fraction being the ratio of the 
total of the weekly fertilities of the plants flowering in that week to the grand total of the weekly fertilities of the whole population over all eight weeks.

When a plant was selected to be a parent, by random choice from all those plants flowering in that particular week, a random test was applied to decide whether the chosen plant should be used as a parent or rejected, the probability of it being used on that occasion being equal to its weekly fertility. Thus a pure A or B flowering for two weeks would be used every time it was chosen, while an $F_{1}$ plant flowering for five weeks (see table 1 ) would be rejected nine times out of ten.

\section{(iii) The starting population}

Initially, the population consisted of 256 pure A plants (16G) and 256 pure B $(16 g)$. Within each subspecies there was variability in respect of the $D$ and $W$ genes, but the two subspecies were identical in their frequency distributions of the possible genotypes, with a mean value for each subspecies of $3 D 3 d 2 W 2 w$; for each of the five loci, the two possible alleles were equally frequent. Thus in the first generation there were always equal numbers of $A$ and $B$ plants in flower at any one time, with a maximum in the central two weeks of the eight.

The first experimental run with this population demonstrated an important oversight. Within a few generations, one subspecies had completely eliminated the other. Although the population size had been kept constant, no provision had been made for maintaining equality of $\mathrm{A}$ and $\mathrm{B}$. Inevitably, chance led to inequality and provided another example of a highly metastable situation with disequilibrium having very strong positive feedback characteristics.

In this situation, the commoner subspecies provides the majority of the pollen, and so is more likely to be pollinated by its own pollen than by that from the other subspecies. For the latter, the reverse is true, and its ovules will be hybridised more often than not. The rarer one subspecies becomes, the less chance does it have of producing non-hybrid seed and the more rapid its decline and elimination. The subspecies in the majority therefore has a selective advantage which becomes increasingly greater.

This is of considerable significance in the more complex experiments to be described later, and will be dealt with more fully then. Here, it was merely a nuisance to be eliminated, and this was done by the occasional addition of $16 G$ or $16 \mathrm{~g}$ plants ( $3 D 3 d 2 W 2 w$ in either case) when necessary to prevent serious disequilibrium, existing plants being replaced at random by these. This modification having been made, the first full run with the pilot model was entirely satisfactory, and will now be described in detail.

\section{(iv) Results}

Fig. $2 a$ shows the constitution of the population after 3 generations in respect of the $G / g$ alleles which characterised the subspecies. There had been massive hybridisation with substantial numbers of $F_{2}$ and backcross progeny. $8 G 8 g$ individuals were the majority class, the total being somewhat greater than the combined total of pure $A$ and $B$.

In fig. $3 a$, the histogram of fig. $2 a$ is broken down to show the number of plants in each genotype which are in flower for each week. The maximum flowering, as at the beginning of the run, was in the centre of the 8-week 
period, with no obvious difference between the subspecies. It must be emphasised that the histograms in fig. 3 represent numbers of plants flowering in each week, not the reproductive potential available during the week; that is, for any particular week a plant flowering during six weeks is represented equally with one flowering for two, provided both are actually in flower during that week.
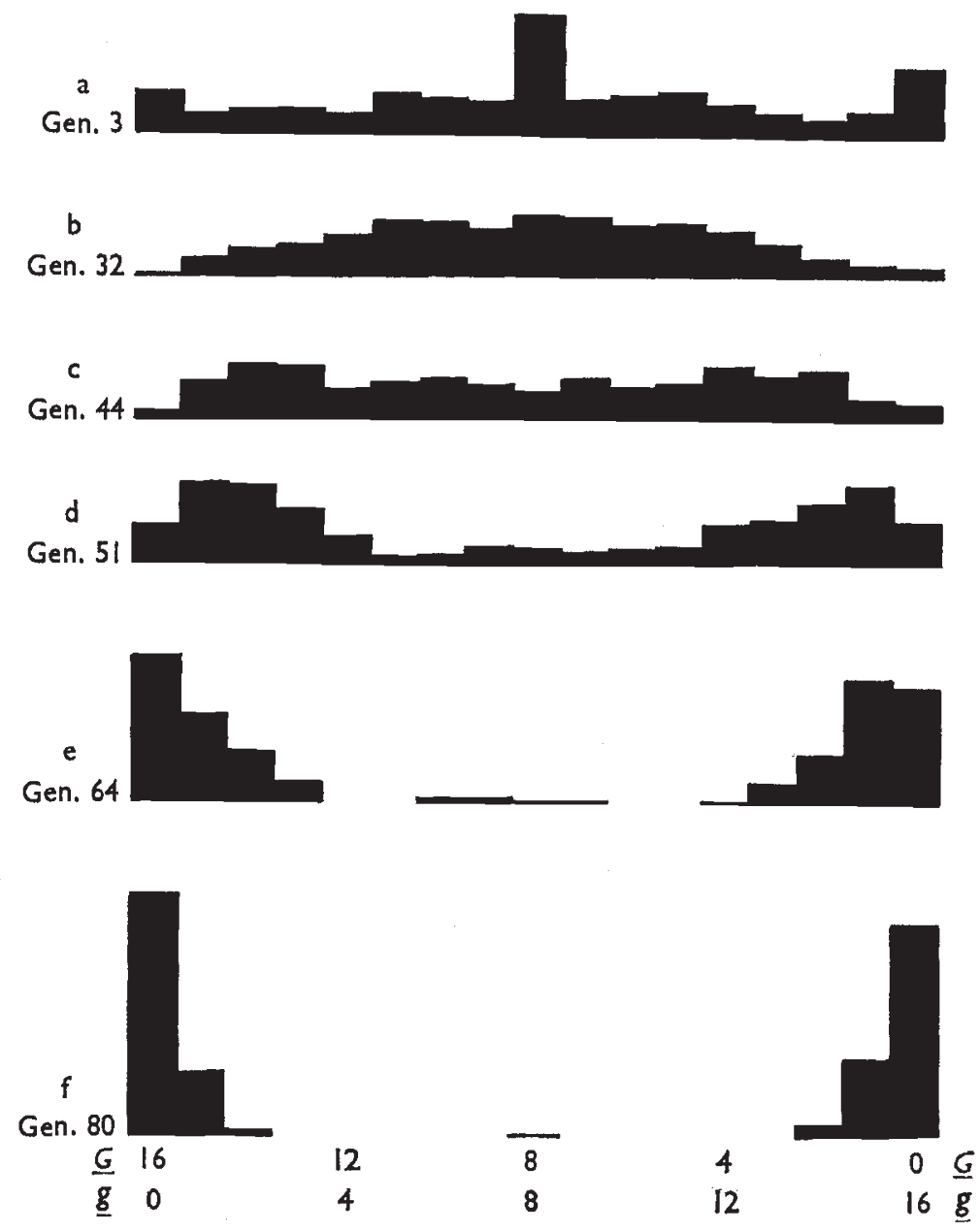

FIG. 2.-The frequencies of the various $G / g$ genotypes at selected illustrative generations of the experiment with the pilot model. The vertical scale is the same throughout; the central peak of section a represents 98 individuals.

After 32 generations, shown in figs. $2 \mathrm{~b}$ and $3 \mathrm{~b}$, the pure subspecies had almost disappeared. $8 G 8 g$ plants were still in the majority, but only just; the central peak was less sharp and the histogram of fig. $2 \mathrm{~b}$ is approximately that of a normal distribution. But when this is broken down by weeks, in fig. $3 \mathrm{~b}$, it is clear that the distribution of the flowering time genes was no longer random with respect to the subspecies-characteristic genes $G / g$. The majority of high $G$ plants were tending to flower during the first three weeks, during which time relatively few high $g$ plants were in flower. The flowering- 


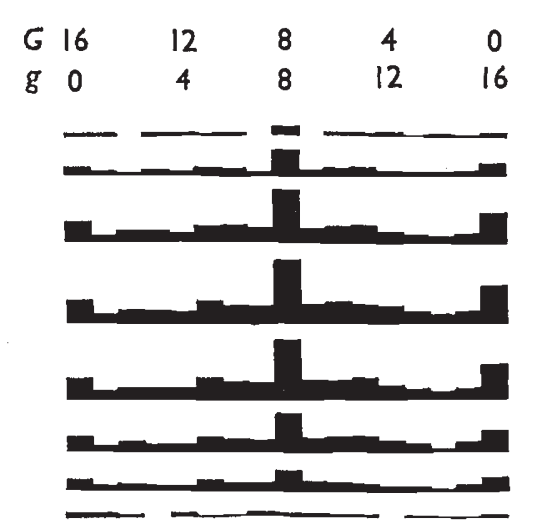

a. Generation 3

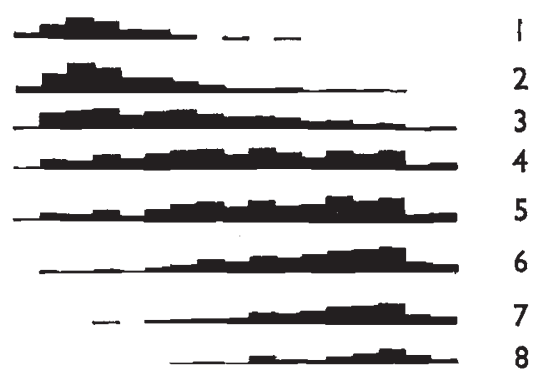

c. Generation 44

$\begin{array}{ccccccc}\text { ¿ } & 16 & 12 & 8 & 4 & 0 & G \\ 3 & 0 & 4 & 8 & 12 & 16 & g\end{array}$

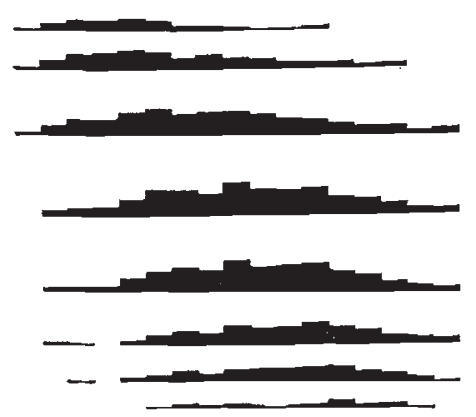

b. Generation 32

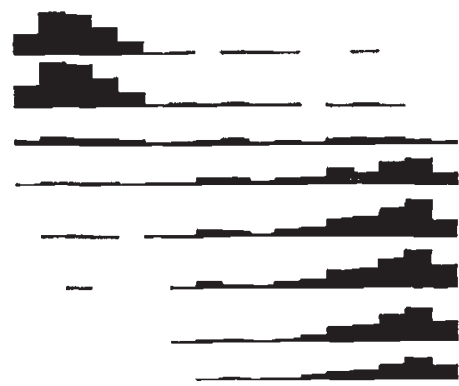

d. Generation 51
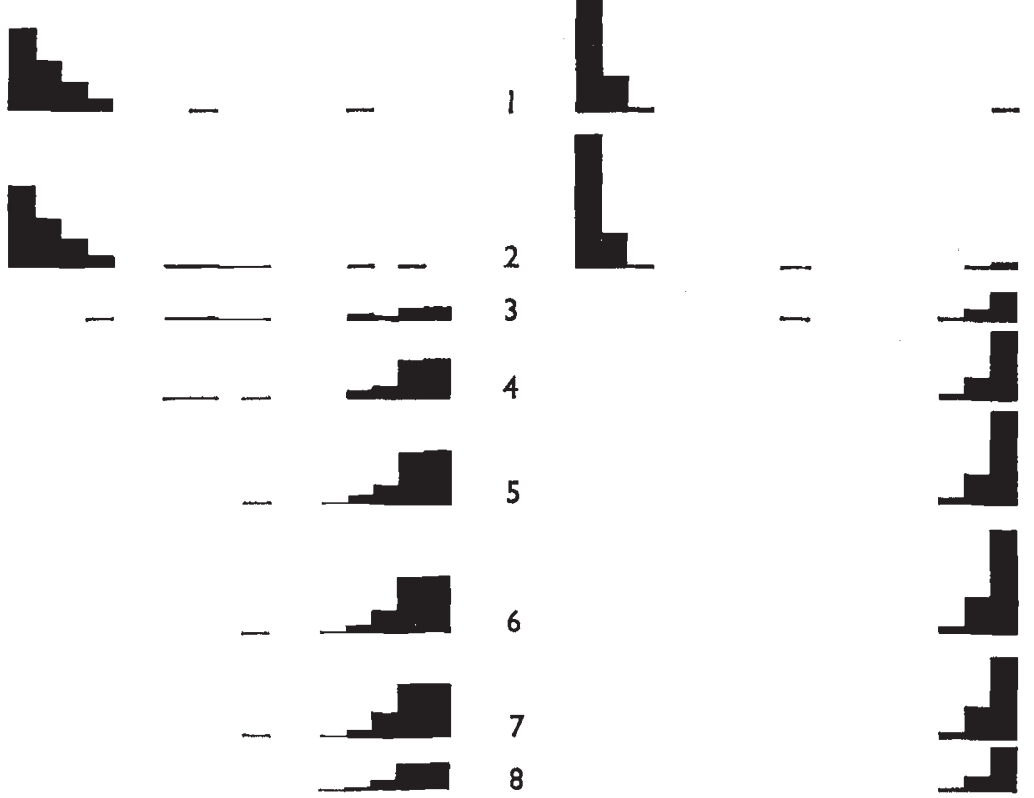

e. Generation 64

f. Generation 80

Frg. 3.-The histograms of fig. 2 analysed to show, for each of the illustrative generations, the frequencies of the different $G / g$ genotypes flowering in each of the eight weeks of the flowering season. The vertical scale is the same throughout; the number of $16 G$ plants flowering in week 1 at generation 80 was 203. 
time shift was more pronounced for the high $G$ plants. There were fewer high $g$ than high $G$ plants, and their flowering was mostly in weeks 3-7. The picture is perhaps clearer if we consider all plants with a majority of $g$, that is all those on the right-hand half of the histograms; these had a peak flowering period in weeks 4-6.

Clearly, by generation 32 the population had acquired potentiality for selection. There was now a distinct advantage to those high $G$ plants which flowered early, since they would have the best chance of avoiding pollination by high $g$ plants with its consequent production of low fertility offspring. There was also a clear advantage to those high $g$ plants which avoided the early weeks, although there was less pressure towards really late flowering.

While it was too early to talk of a period of transition in flowering from $A$ to $B$, the indications were that there would be an asymmetry in the flowering of the subspecies. Fig. $3 \mathrm{~b}$ suggests that a transition period was developing between weeks 3 and 4, rather than halfway through the total period available for flowering. This was presumably an effect of chance in a small population (as indeed was the origin of any difference in flowering time), but it does suggest that there was no strong pressure within the system towards symmetry.

Other effects probably arising initially by chance were the rather higher proportion of high $G$ (tending towards purity of A) than high $g$ plants in the population, and the greater tendency towards isolation in flowering time of high $G$ plants; once these effects had arisen, they might be expected to augment one another, and they would then be more noticeable in later generations.

By generation 44 there was a striking change in the picture. Fig. 2c shows that $8 G 8 g$ plants are no longer in the majority, and the histogram is clearly bimodal. The greater approach to purity of $A$ than of B was now very noticeable, the peak at the A end being in the region $14 G 2 g$ to $13 G 3 g$ with the maximum at $14 G 2 g$, while the peak at the B end goes from $4 G 12 g$ to $2 G 14 g$ with the maximum at $4 G 12 g$.

The explanation of the change in form of the histogram is clear from fig. 3c, which shows that high $G$ plants had made considerable progress towards flowering-time isolation, and therefore towards reduction in hybridisation. Not only did they show no more than a moderate overlap in flowering time with high $g$ plants, but during their first two weeks of flowering they were scarcely overlapping the central hybrids (plants in the range $10 G 6 g$ to $6 G 10 g$ ) which did not come strongly into flower until week 3 . High $g$ plants on the other hand, flowering strongly over the last five weeks (as contrasted with the first three weeks which had most of the flowering of high $G$ plants), overlapped the flowering time of the central hybrids considerably. A consequence of this was the slower approach to purity of $B$, as seen by the relatively lower proportion of high $g$ plants.

The transition period of flowering in generation 44, though less vague than it had been in generation 32, was still not clear. But by seven generations later, generation 51, it had become sharply defined. At the same time, and as a consequence, the number of central hybrids had become drastically reduced (fig. 2d) and both subspecies had made considerable progress towards purity. The high $G$ side maintained its lead in this respect, although both showed maxima at corresponding points, $15 \mathrm{Gl} g$ and $1 \mathrm{G} 15 \mathrm{~g}$.

In generation 51 the flowering of high $G$ plants was almost entirely 
confined to the first two weeks (fig. 3d), while that of high $g$ was spread almost entirely over the last five. The only significant overlap between the two subspecies, as we may now begin to refer to them again, was in week 3 . This week was particularly interesting, for although approximately equal numbers of high $G$, central hybrids and high $g$ were flowering at the same time, these numbers were very small. Indeed, there were fewer plants flowering during week 3 than in any other week except week 8 .

By generation 64 (figs. $2 \mathrm{e}$ and $3 \mathrm{e}$ ), $\mathrm{A}$ and $\mathrm{B}$ were almost isolated from one another though still some way from purity. For A, $16 G$ plants were clearly in the majority, but for B $1 G 15 g$ had a slight majority over $16 g$. High $G$ plants were almost completely confined in their flowering to the first two weeks; their date of flowering genes were completely fixed, all being $6 D$, and their length of flowering genes were almost completely fixed, all being $4 W$ except one $13 G 3 g$ which was $3 W 1 w$. By contrast, there was still a great deal of $D$ and $W$ variability among high $g$ plants.

During the first week, there was one hybrid and one $3 G 13 g$ plant flowering with 254 high $G$ plants; in the second week, six central hybrids and four high $g$ plants were flowering with 254 high $G$ plants; in the third week, one $13 G 3 g$ plant was flowering with six central hybrids and 46 high $g$ plants. That was the extent of the overlap and of the potentiality for hybridisation. The total number of central hybrids in the population was six (in the following generation it was only three). The evolution of genetic isolation was practically complete.

During the next 16 generations, to generation 80 , the only important change was the continuing advance to purity of $\mathrm{A}$ and $\mathrm{B} ; 16 \mathrm{G}$ and $16 \mathrm{~g}$ plants were now very much in the majority, with the former maintaining their lead (fig. 2f).

The somewhat greater overlap in flowering time shown in fig. 3f, with a few B plants flowering in the first two weeks, is largely fortuitous. While B retains genetic variability, there will always be a tendency for some plants to flower while $\mathrm{A}$ is still flowering, and the numbers of these will fluctuate from generation to generation.

The presence of only one hybrid in the population at generation 80 (at which point the experiment was stopped), though excellent for the purposes of illustration, was even more a matter of chance, of which there were two sources. One was the fluctuating but small number of $\mathbf{B}$ plants flowering in weeks 1 and 2. The other arose from the balancing operation in which pure A or B plants were added in order to maintain equality of numbers. All added plants flowered during weeks 3-6. B plants added in this way would by now find themselves with B plants and hybrids only, and would have no effect on hybridisation. But any A plant so added would flower only with B plants and hybrids, and any other A added at the same time. This would be expected to lead to the formation of hybrids, and could also temporarily upset the fixation of $D$ and $W$ in A plants, which was complete in generation 80 .

The actual numbers of central hybrids in the population varied considerably between generations 64 and 80, with a value of 2 at generation 67, a maximum of 21 at generation 71 , and minimum values of 1 at generations 79 and 80 . The maximum value followed three generations in which $A$ plants were added, but from generation 71 onwards, only B plants were added except for a single $\mathrm{A}$ in generation 74 . 
A particularly striking feature of the results, which could be seen developing gradually as far back as generation 32 , was the fixation of $W$ and $D$ in $\mathrm{A}$, with A plants occupying the minimum possible flowering period at the earliest possible flowering time, while $B$ plants retained substantial variability and made full use of the six weeks left vacant by $A$, the only check being against too much overflow into week 2. Genetic fixation at the $W / w$ and $D / d$ loci was not to be expected in both species, as A and B had now become. There was more genetic variability available at those loci than was required for the separation of $\mathrm{A}$ and $\mathrm{B}$. If $d$ and $W$ had become fixed in $\mathrm{B}$, there would have been a period of four weeks in the middle during which nothing flowered. Once the two species had come to be non-overlapping in flowering time, there was no selection pressure to force them farther apart, and a nonflowering period could only have come about by chance fluctuation in gene frequency.

The extent of the variability retained by B is shown in figs. 4 and 5 . For the date genes in $\mathrm{B}$ there is no clear maximum in the class of latest flowering, $1 D 5 d$ and $6 d$ being equally numerous. For length of flowering, the maximum is at three weeks, $3 W$ l $w$, with two weeks the second smallest class. The peak flowering period for $\mathrm{B}$ is weeks 5 and 6 .

Comparison of figs. $3 \mathrm{e}$ and $3 \mathrm{f}$ might suggest that $\mathrm{B}$ was tending to flower slightly earlier at 80 than at 64 generations, and that perhaps there may have been some pressure forcing $\mathrm{B}$ as far in the direction of early flowering as was compatible with the avoidance of hybridisation. To draw this conclusion from those figures would be unjustified. Between generations 64 and 80 there was considerable fluctuation from one generation to the next in the numbers of high $g$ individuals in the various date and length of flowering classes, and there is no reason for drawing any other conclusion than that generation 80 was one in which by chance there was a slightly higher number of early flowering B plants than in generation 64 .

It is difficult to decide whether the pattern of flowering-period compression of one species and much longer period of the other is primarily a property of the model or whether it has any significant relevance to natural populations. It was not a chance occurrence of the one experimental run, for the same thing happened in a second run with the same model.

There is no reason why the transition in flowering from $A$ to $B$ should develop precisely in the middle of eight weeks. There is plenty of scope for chance to affect this. Once an asymmetry has developed, it would be expected to increase. The subspecies which withdraws most rapidly to one end or other of the flowering period is not only going to avoid hybridisation with the other subspecies, it will also tend to avoid pollination by hybrid plants (using this term loosely to include $F_{2}, F_{3}$ etc. segregates), which were at maximum flowering during the central part of the eight weeks. This would not only lead to more rapid reversion to subspecific purity, but would mean more rapid evolution of change in flowering time, since dilution of early flowering $D$ alleles through crossing with hybrids (as well as with the other subspecies) would be diminished.

It would have been possible to have constructed the model in such a way that the pattern shown by fig. $3 \mathrm{f}$ would be much less marked, or even nonexistent. The introduction of a slight disadvantage in very early or very late flowering, and a slight fall in total reproductive capacity with shortening of the flowering period, both of which might reasonably be expected to 
occur in a real situation, would probably have this effect. These would have first-order selection pressures, whereas selection of flowering-time alteration through hybridisation avoidance is a second-order effect; that is, selection is primarily against hybrid infertility, and is only effected indirectly

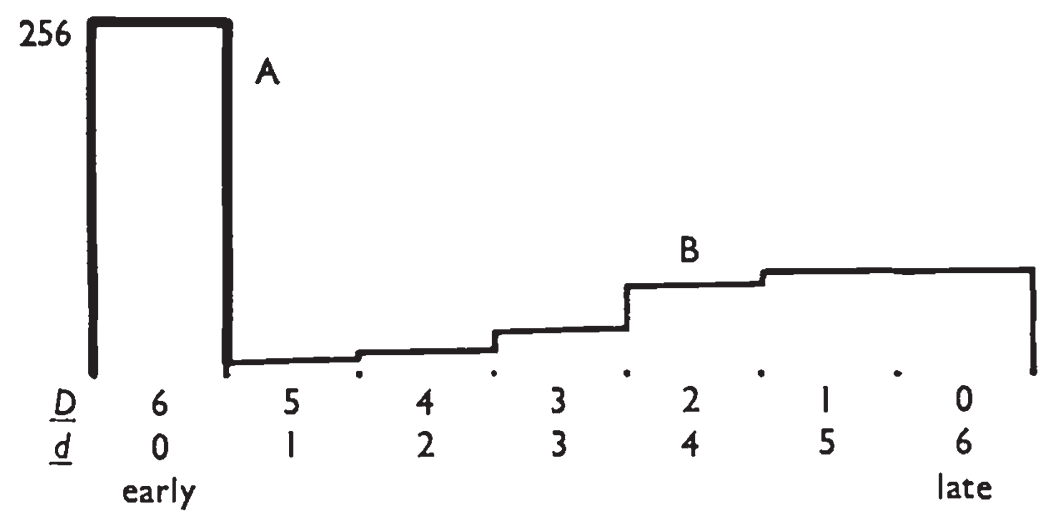

Fig. 4.-Frequency distributions of flowering-time genotypes $(D / d)$ shown separately for A $(16 G-14 G 2 g)$ and $B(2 G 14 g-16 g)$ at gencration 80 of the pilot experiment.

through flowering period. Second-order effects are of much less magnitude than the effects of first-order selection (Crosby, 1963), and slight modification to the model might well have had a substantial effect on the final pattern of flowering time of the species.

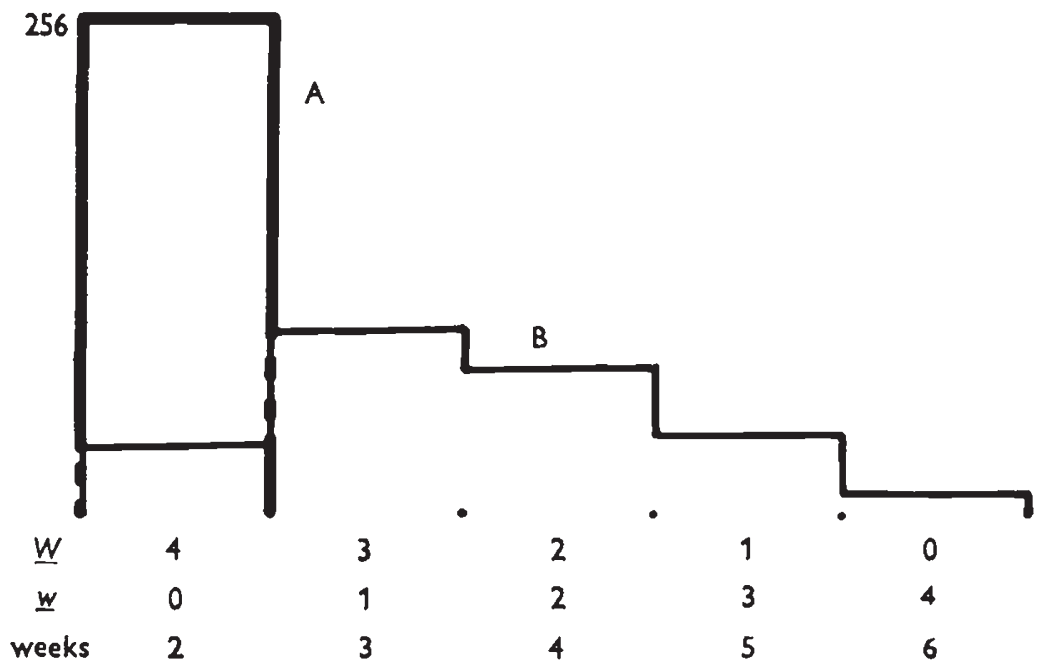

Fio. 5.-Frequency distributions of the length of flowering-period genotypes $(W / w)$ shown separately for $\mathrm{A}(16 G-14 G 2 \mathrm{~g})$ and $\mathrm{B}(2 G 14 \mathrm{~g}-16 \mathrm{~g})$ at generation 80 of the pilot experiment.

Nevertheless, it is of interest that essentially the same pattern (but with late and early reversed) is shown by the two species which were in my mind at the start of this work. In England at least, Primula vulgaris which flowers early has a long flowering period, both as a species and as an individual plant. 
The later flowering $P$. veris flowers for a shorter time as a species, and individual plants also complete their flowering more rapidly. This will be referred to again in a later section.

I decided not to elaborate this pilot model in the way outlined, though in a more complex model the relation between length of flowering and total reproductive capacity was considered, and will be discussed later. Although unrealistic in several ways, these preliminary experiments clearly demonstrated both the feasibility of the application of computer simulation techniques to the study of the evolution of genetic discontinuity, and the theoretical justification of the belief that such discontinuity could arise through selection against hybridisation.

The results of the pilot model have been presented in considerable detail, because they provide a generalised picture of a process which is easier to describe and follow in the simple situation, and form a good basis for the account of the more complex model which follows.

\section{The POPUlation genetics of AN INTERFAGE}

The pilot model imagined a small population in which two subspecies had come together, completely interpenetrating and thoroughly intermingled. Much more interesting and realistic is the situation when the subspecies first come into contact, and interpenetration is confined to a narrow zone which may be referred to as the interface between them.

In one way, the pilot model had something of this situation in that the compensating plants added in order to prevent the development of imbalance between A and B could be regarded as having come from adjacent pure populations of A and B. But this immigration was entirely artificial and the numbers of such plants coming into the model population were determined solely by the requirements of the model. In the models which are about to be discussed, such immigration, producing a zone of interpenetration, was an entirely natural occurrence within the model, and no attempt was made to counter any imbalance between A and B which might have developed. That artificial element of the pilot model was thus entirely discarded, but the discussion of why it was necessary there is of considerable relevance to the new situation. It will be remembered that the fundamental point was that, other things being equal, in a situation where two subspecies (or species) are hybridising with the production of infertile hybrids, the minority subspecies is always at a disadvantage and will decline to ultimate elimination.

The relevance of this to the new situation can be seen if we first visualise the general form of the model population which is to be considered. This introduced a new concept, of space, position and distance. The area occupied by the two subspecies at the beginning of an experiment was imagined to be a long narrow rectangle, transversely halved so that initially $\mathrm{A}$ was evenly distributed through one half, B similarly in the other half, with A and $\mathrm{B}$ in contact but not interpenetrating. Gene flow within this area depended on the movement of pollen and seed, which had now to be restricted, otherwise the position would have been essentially the same as in the pilot model. There had of course to be some movement of pollen and seed, and this allowed hybridisation through pollen movement across the interface, and through the passage of seed from one subspecies into the territory of the other, turning the interface into a zone of interpenetration. 
The question arose of how broad this zone would be, and whether it would grow, with increasing interpenetration of $\mathrm{A}$ and $\mathrm{B}$. So long as hybridisation between $\mathrm{A}$ and $\mathrm{B}$ was possible, significant interpenetration seemed to be unlikely; for example, any A plants finding themselves through seed dispersal in the territory of $\mathbf{B}$, or $\mathbf{B}$ plants in the territory of $\mathbf{A}$, would be in the minority position already discussed and subject to the disadvantage of greater liability to hybridisation. The further the penetration, the greater the liability, which would thus be expected to act as a powerful factor against invasion; the zone of interpenetration would be expected to remain a narrow one so long as hybridisation was easily possible. But if, as in the pilot experiment, there was evolution of a difference in flowering time in the region of the interface, then there would be inhibition of hybridisation, interpenetration would accordingly become increasingly possible, and the zone should broaden.

The principal disadvantage to a very penetrant invader might then be a reduction in seed set resulting from its being out of range of an adequate supply of pollen of its own subspecies; the extent of this disadvantage would depend on how far self-fertilisation was possible.

With pollen and seed dispersal, as well as numbers of A and B, subject to random fluctuation, there would be variation both in penetration pressure and resistance through hybridisation. This might lead both to fluctuations in the extent of interpenetration and to movement of the zone. As will be explained later, the model allowed variation in plant density within the population and if, say, there came to be more A plants near the interface than there were $B$ on the other side, then A might exert a pressure on the interface which would cause this to move into B's territory.

Selection of genetic barriers to hybridisation can only take place where $\mathrm{A}$ and $\mathrm{B}$ are in contact. This meant that the number of plants actually involved in the process of selection would be much smaller than in the pilot model, unless either the model population was very large or the permitted movement of pollen and seed was such that the zone of interpenetration would quickly come to occupy a large proportion of the area. Population size was limited by consideration of size of computer store, and of time taken by the programme. Too large an early zone of interpenetration would have meant too close an approach to the conditions of the pilot model. Through trial and error, an acceptable compromise was reached which will be described in more detail later. This involved rather small numbers within the breeding range of any one plant, and consequently there was rather a large effect of random variability; in practice, this proved to be tolerable.

If selection could only take place in the zone of interpenetration, the question then arose as to how quickly and how far, if at all, the selected alleles could move away from the interface and outwards through the pure subspecies, and whether a selected genotype could become established in a subspecies well away from the zone of interpenetration. With a difference in gene frequency between distal and interface ends of a subspecies, and selection operating only at the interface end, then ordinary gene flow within the subspecies might be expected to cause the advantageous alleles to flow towards the distal end, and their frequency consequently to increase even well away from the site of selection.

However, in repeating with approval the opinion of Moore (1957), Mayr (1963, p. 549) says, "the genetic factors responsible for reproductive 
isolation should, on this theory, be restricted to zones of overlap between the related species, since there would be no selective advantage to have these ad hoc mechanisms spread beyond the area where they are favoured by selection ", and adds that there is no evidence that isolating mechanisms are geographically thus confined.

It was hoped that the new model might throw some light on this question.

\section{(i) The model}

In its basic assumptions, the new model followed generally the pilot model. Apart from the introduction of a small starting difference in flowering time between the subspecies, the two models were genetically identical, including the determination of weekly and total fertility. The only considerable changes were those primarily or consequentially associated with the introduction of dimension, and in population size.

The model itself went through a series of evolutionary stages, as simulation procedures were modified and biological nonsense eliminated. This was a lengthy process, as apparently trivial features of the model, introduced without sufficient preliminary thought, sometimes produced results which were nonsensical. For example, one early version with an apparently reasonable method of simulating pollen transport succeeded in eliminating all plants, of both subspecies, from the centre of the population. One particular difficulty lay in making sure that there was no fortuitous selection irrelevant to the problem under investigation. As has already been pointed out, the selection of barriers to interbreeding is small in magnitude; the existence of quite small irrelevant selection pressures on the genes involved could easily lead to misleading results. For example, the weekly fertilities could not always be expressed exactly in a fixed number of binary digits, but had to be expressed as rounded approximations; this led, variously, to slight selection pressures for or against the length of flowering genes $W$, which clearly had nothing to do with selection against interbreeding. In all cases where rounded approximations had to be used, this was arranged in such a way that selection was minimised and was in a direction contrary to that expected from selection against interbreeding.

A detailed account will be given later of the results of the first wholly satisfactory run (run 16). The description of the model about to be given refers specifically to the model used in that run. Other experiments will be described by reference to this basic model.

The population occupied a long narrow rectangle, divided transversely into 256 equal rows, as suggested in fig. 6. Each row had spaces for 15 plants. At the start, the plants were distributed evenly through the population, each row having 10 plants. The subspecies each began with 1280 plants occupying half the population area, being in contact at the centre of the population but with no mixing. There was no attempt artificially to maintain equality of numbers of $\mathbf{A}$ and $\mathbf{B}$ once any experimental run had started, but the total population size was maintained at about 2560 . Since each row had spaces for 15 plants, but on average only 10 spaces per row were occupied, variation in plant density from 0 to 15 plants per row was possible anywhere in the population.

The pilot model, which had started with the two subspecies flowering at identical times, had demonstrated that random fluctuations could lead to a flowering-time difference sufficient to initiate a process of selection. 
I felt justified in assuming the validity of this demonstration, and decided that this model should start with a small difference in flowering time between $A$ and $B$. Since selection could only take place in a relatively small fraction of the population, around the interface, it seemed likely that it would be a much slower process than in the pilot model. The number of generations likely to pass before random development of a flowering-time difference was obviously unpredictable, and might be very many; there seemed to be no point in possibly wasting a great deal of computer time over something which was unlikely to give any new useful information.
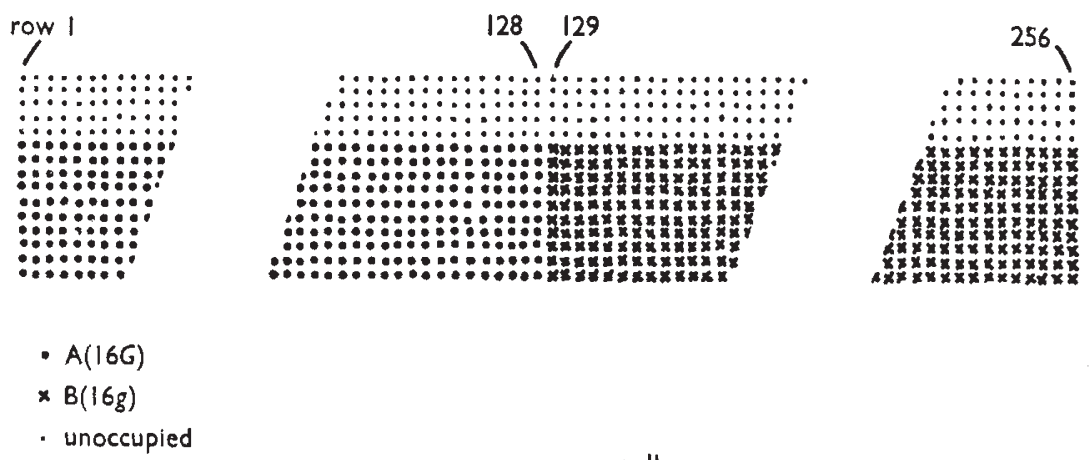

- $A(16 G)$
$\times B(16 g)$
- unoccupied

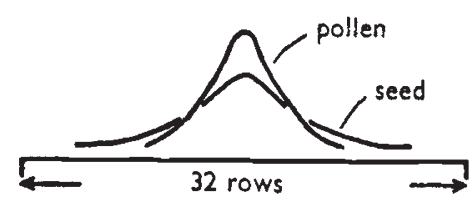

Fic. 6.-Diagram to show layout of the starting population of run 16. There are 256 rows; each of the leftmost 128 rows contains 10 plants of pure $A(16 G)$ and 5 vacant places; each of the rightmost 128 contains 10 plants of pure B $(16 \mathrm{~g})$ and 5 vacant places. At the bottom are shown distribution curves indicating the range of seed dispersal and pollen transport; these curves are drawn to the same horizontal scale as the population diagram which, had it not been fragmented, would have been 3.4 times as long as it appears here.

In order that each subspecies should be genetically homogeneous at the beginning of each experiment, all rows of a subspecies were genetically identical, genetic variation occurring within the ten plants of each row. For the whole population, each row contained two $1 W 3 w$ plants, six $2 W 2 w$ and two $3 W l w, w$ frequency being 0.5 at each locus. The two subspecies were thus identical in respect of length of flowering period, averaging $2 W 2 w$ as in the pilot experiment and having identical variability. For the date of flowering genes, of which the $D$ alleles promote early flowering, each row of subspecies A had two $2 D 4 d$, four $3 D 3 d$, three $4 D 2 d$ and one $5 D 1 d$, the frequency of $d$ at each locus being 0.45 . For $\mathrm{B}$, each row had one $1 D 5 d$, three $2 D 4 d$, four $3 D 3 d$ and two $4 D 2 d$, the frequency of $d$ at each locus being $0 \cdot 55$. The difference in the means for the two subspecies would correspond to a difference in the time of maximum flowering of about half a week; the amount of genetic variability was again the same for each subspecies. A visual description of the starting condition of the population is shown by the three sections of fig. 7, and this form of presentation will be used to illustrate subsequent evolutionary progress. 
The difference between the flowering of $\mathrm{A}$ and $\mathrm{B}$ ten generations from the start of run 16 is illustrated in fig. 8, which shows for the whole population the numbers of $\mathrm{A}$ and $\mathrm{B}$ in flower in each week; by this time, the genotype frequencies had settled down after the somewhat arbitrary starting structure,
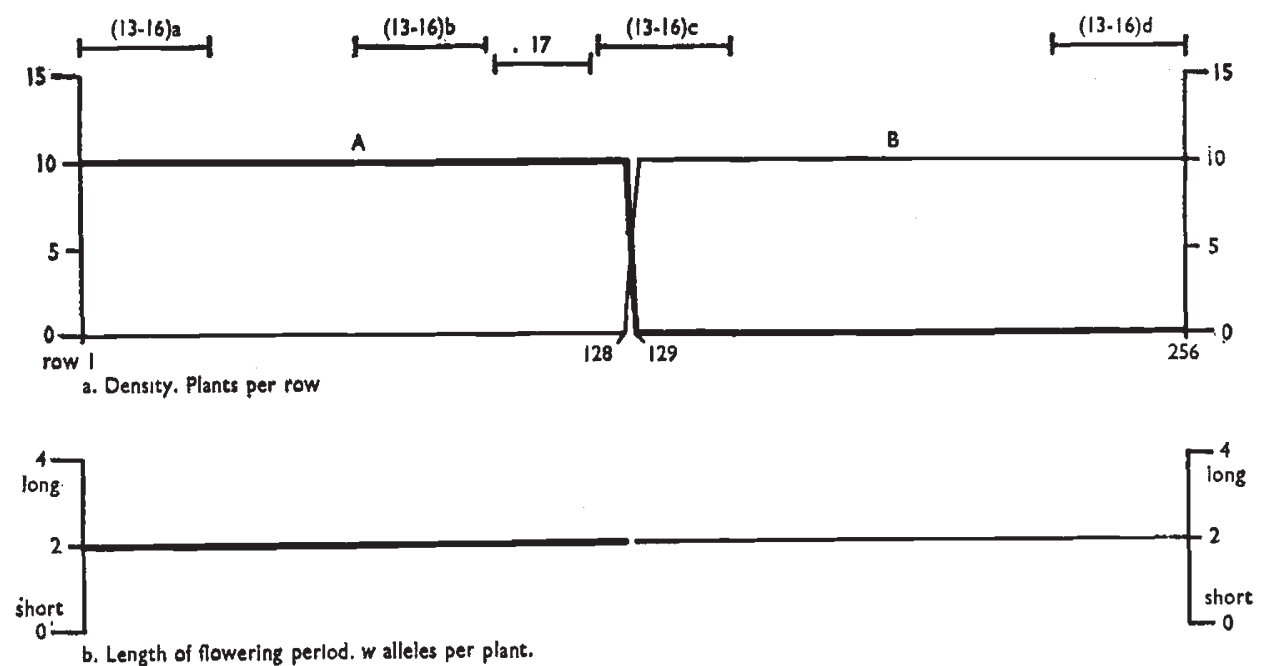

b. Length of flowering perlod. $w$ alleles per plant.

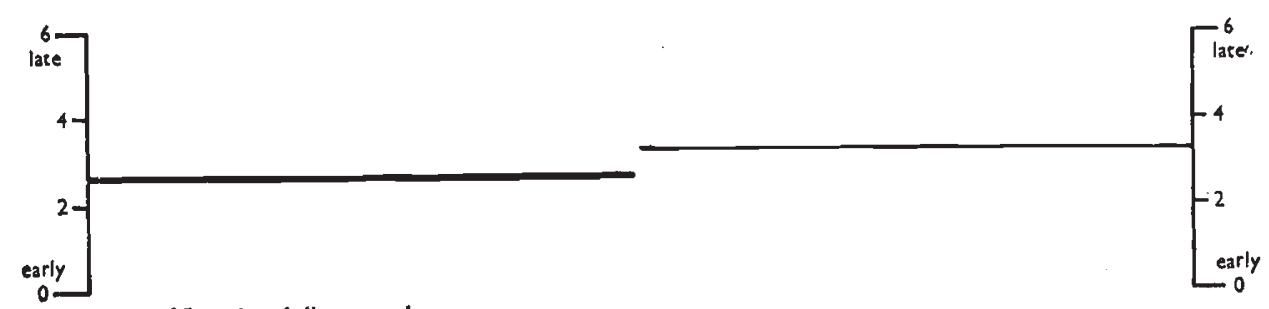

c. Date of flowering. \& aileles per piant

FIG. 7.-Diagrams to show starting condition of the population of run 16. This legend also serves to explain figures $9-12$ and 18. Each section represents the full length of the population. Section a shows the plant density of each subspecies (A=16G-14G2g; $\mathrm{B}=2 G 14 \mathrm{~g}-16 \mathrm{~g}$ ) as plants per row; in figs $9-12$ and 18 , hybrids $(10 G 6 g-6 G 10 \mathrm{~g}$ ) are represented at the base of each section a by dots, each representing a hybrid and indicating the row in which it occurs. Sections b and $c$ show respectively the mean number of $w$ alleles and $d$ alleles per plant of each subspecies, calculated in adjacent sections of 2 rows; these lines of fig. 7, representing the starting gene frequencies, are repeated as thin lines in figures 9-12 and 18 to give an indication of change in gene frequency. At the top of section a in this figure are indicated the sections of the population which are represented by the histograms of figs. 13-16.

and fig. 8 gives a better picture of the situation than histograms of the actual start would have done, and shows that the difference in flowering period between $\mathrm{A}$ and $\mathrm{B}$ was much less than the difference shown by the pilot experiment after 32 generations (fig. $3 \mathrm{~b}$ ).

(ii) The simulation of position and distance

We come now to the questions of pollen transport and seed dispersal, and the way in which these were simulated. Seed dispersal presented fewer problems and will be described first. 


\section{(a) Seed dispersal}

Broadly speaking, seed dispersal was normal about the row of origin of the seed. The row in which a seed was to land and produce a new plant was determined by random reference to a table containing zeros and small positive and negative integers in frequencies corresponding to a distribution which was exactly symmetrical and approximately normal around a mean of zero. The selected number was added to the number of the row of origin, and the total gave the number of the row to which the seed was to be dispersed. Half the seeds fell within a range of three rows on either side of the

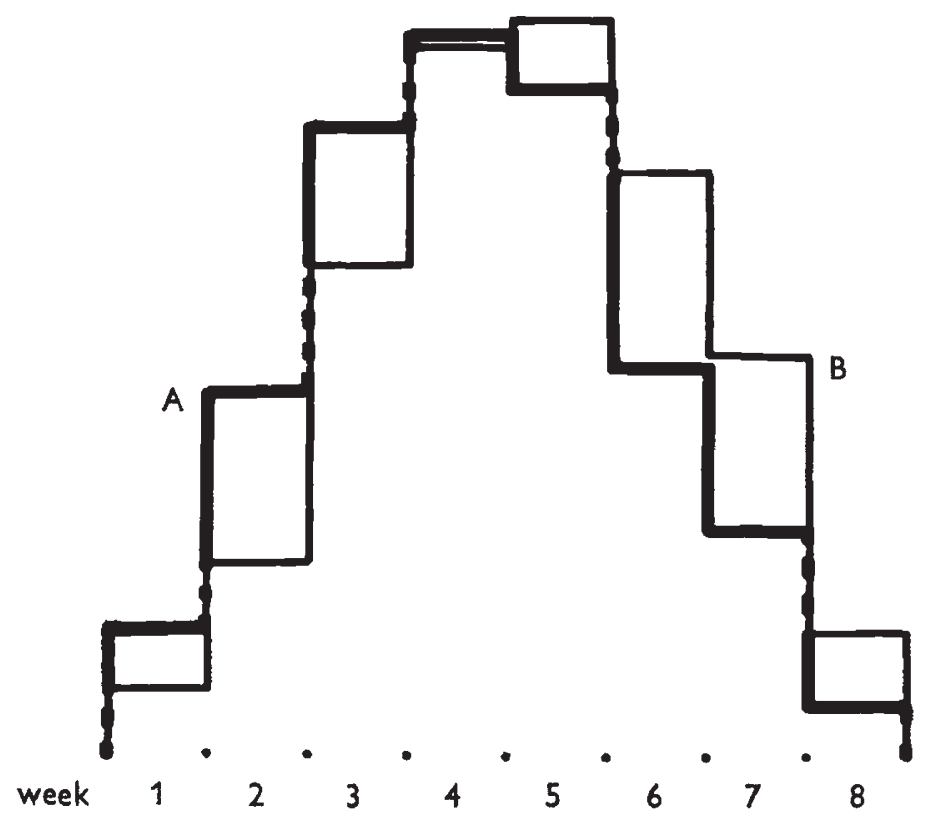

Fio. 8.-The flowering periods of the two subspecies over the whole population after 10 generations of run 16 , shown as the number of plants of each in flower in each week. The highest point on the $\mathrm{B}$ histogram represents 1108 plants. Where the lines of $\mathrm{A}$ and $B$ coincide, a line of alternating thickness is used.

row of origin; the maximum dispersal was 12 rows and the mean was 4.25 rows, which was approximately one-sixtieth of the total length of the population.

In applying this routine for seed dispersal, two problems arose. Firstly, the resultant row number might indicate a row outside the population limits, corresponding to a situation which might well arise naturally. In early runs, seeds falling outside the population had been treated as lost, and initially these could be as many as 20 per annum from each end of the population. This led to a reduction in the number of adult plants below the 2560 at which it was desired to maintain the population. To prevent a decline in population size, the reduced number of plants had to produce the full number of 2560 seeds, and the consequent slight increase in reproductive rate was spread evenly through the population (any other procedure would have involved considerable complication of the programme and an excessive amount of time). This meant that there was a slow draining off of genes 
from the ends of the population, and consequently a small centrifugal gene flow which was due entirely to the construction of the model and not at all to any selective process. Since one of the objects of the experiments was to see whether there was any centrifugal gene flow arising from the biology of the system, it was essential to eliminate as far as possible any irrelevant factors which might themselves cause such gene flow. Heavy seed loss from the ends of the population had therefore to be eliminated, and the method chosen was to repeat, from the row of origin, the seed dispersal routine for any seed falling outside the population either until it fell inside or until five unsuccessful attempts had been made.

This was not an entirely satisfactory way out of the difficulty. It could be regarded approximately as simulating a situation in which the plants extended beyond the limits of the experimental population but with essentially the same average genotype as those just inside the population, as many seeds coming from outside into the population as were lost.

The second problem arose from the possibility that the row chosen to receive the seed might already have its full quota of 15 seeds. Where too many seeds were allotted to any row, the number was reduced to 15 by randomly discarding the appropriate number of seeds from those originally allotted to the row. This method was preferred to the "try again " routine used in the case of terminal loss, since that would have tended to inflate numbers in adjacent rows. The method used provided a slight check against excessive density, the origin of which was in any case largely fortuitous.

\section{(b) Pollen transport}

The treatment of pollen transport was more complicated than that of seed dispersal, since it involved more assumptions about the biology of the situation. For each seed, the reproductive process began with the selection of a seed parent, followed by selection of a pollen parent from among the plants flowering in the same week. The method of selection of the pollen parent involved several assumptions about the behaviour of pollinating agents. Little enough is known about the behaviour of real pollinating agents. A hypothetical one which is supposed to represent generalised pollinating behaviour is no easier to deal with. The computer tends to become the master here, for any deviation from simplicity in simulation would lead to programming complexity and considerable increase in running time; it was necessary to balance any advantage from more precise simulation (which would in any case be based more on guesswork than on knowledge) against the smaller number of generations which would be produced in a given running time. The aim had to be simplicity provided it was biologically sensible.

The following is an example of the kind of problem which arose. Any plant chosen as seed parent will almost always have a greater density of flowering plants on one side of it than on the other. ("Flowering plants" means plants actually in flower at the time which is being considered.) The distribution of flowering plants about the seed parent will rarely if ever be symmetrical, and the nature of the asymmetry will differ according to the row in which the seed parent occurs, from one week to the next and from one generation to the next. Selection by simple reference to a single permanent distribution table, as in the case of seed dispersal, is therefore not 
possible, while the use of many tables constructed to fit many different possibilities is quite impracticable.

Before deciding on the method of selection, a decision had to be made as to whether pollen was equally likely to come from either side of the seed parent irrespective of any difference in flowering plant density between the two sides, or whether it was more likely to come from the side on which more pollen parents were available; the latter would require a decision on the likely relation between flowering plant density and chance of providing the pollen parent. In the lack of adequate knowledge, it seemed both simple and reasonable to assume that there was such a relation and to make it one of direct proportionality.

The density was determined as the number of flowering plants in the seven rows adjacent to the chosen seed parent on the side in question (or between the seed parent and the end of the population when this was less than seven rows away). If there were $a$ plants to the left and $b$ to the right, the chances of pollen coming from left or right were $a /(a+b)$ and $b /(a+b)$ respectively, and were realised by the use of pseudo-random numbers. No direct account was taken of any possible difference in weekly fertility, but since each selected potential pollen parent was subjected to a fertility test, the side from which the eventually used pollen parent came was on average decided approximately on the basis of total reproductive capacities for the week in the seven-row regions on either side of the seed parent.

The next problem was that of the distance from which the pollen might come. It might be expected that the denser the flowering plants the shorter the individual flights of the pollinating insects from flower to flower, and that there might be a tendency towards a shorter mean distance of pollen transport on the denser side. In nature, this would depend to some extent at least on the habits of the pollinating insects, but it is not simply a matter of how long was the last flight before the critical pollination. The insect will carry pollen from a number of visited flowers, and many factors will influence the distance over which pollen from a visited flower will remain on the insect.

In order to choose which plant was to supply the pollen, the plants in the appropriate seven rows were imagined to be in a single line, as though they had been taken (referring to fig. 6) in order from the bottom of a row to the top and thence to the bottom of the next row on the right, and so on. Plants below the seed parent in its own row would be considered as those closest to it on its left, plants above it as those closest to it on its right. Reference was then made to a table in which relative distances (from the seed parent) occurred with frequencies corresponding very roughly to onehalf of a normal distribution curve with zero as its mode and its end corresponding to the position of the most distal plant in the imaginary line. A random selection from the table indicated a point along the line of potential pollen parents (left or right of the seed parent as already decided) and the flowering plant nearest this point was chosen.

This procedure meant that while, as between the two sides of the seed parent, there was no relationship between mean density and average range of pollen transport, there was an effect of density on any one side, since high density near the seed parent would produce a shorter mean distance of pollen transport, high density away from the seed parent a longer distance. There was perhaps some inconsistency here. This could have been avoided, 
but at the expense of an increase in running time; the subroutine selecting pollen parents was much the most complex and time consuming of those involved in the process of reproduction, and was used more than 2500 times per generation. There were several places at which simulation might have been improved by programme elaboration, but such improvements would always have involved an element of guesswork about biology, and had they all been introduced could easily have doubled the running time of the whole programme. There seemed little likelihood that the simplifications which were left in the model would have any significant effect on the answers to the questions which were being asked of the model, and so long as they were clearly understood it seemed better to retain them than increase the running time.

The way in which the distribution curve was applied did not allow choice of pollen parents to be attempted beyond the actual area within which flowering plants occurred, so attempted selection beyond the ends of the experiment did not in this case arise. Half the pollen parents came from within a range of 1.7 rows on either side of the seed parent; the mean distance was 2.3 rows and the maximum range was seven rows. Gene movement by pollen was thus less than by seed.

Another question which had to be considered was whether a plant's chance of setting seed was affected by the density of flowering plants around it, or by their fertility. My own experience of a number of species suggested that unless plants are really isolated they usually manage to set plenty of seed, and that there is not a great deal of effect of plant density on seed set; at least until the density becomes very low. Emerson (1939) found that apparently isolated plants of the self-sterile Oenothera organensis in New Mexico set ample seed. Plants capable of self-fertilisation would be expected to suffer little loss of reproductive capacity in isolation. It was therefore assumed in this model that the quantity of seed set by a plant was independent of the number of pollen parents available; if there was no other flowering plant within the seven-row range, then the plant was self-fertilised.

I felt, however, that low average fertility of the available pollen parents might have some effect, for example if bad pollen on the stigma provided an obstacle to good pollen. This was taken into consideration, though not very strongly, in the following way. When each potential pollen parent was chosen, it was tested for fertility; if it failed the test, it was rejected and a further choice made, up to a maximum of three tries for any one choice of seed parent. If three potential pollen parents failed the fertility test, the chosen seed parent was discarded and a new choice made. To that extent there was a small lowering of reproductive capacity of all plants, hybrid or not, in areas with a high proportion of hybrids, in addition to any that may have been due to the genotypes of the plants themselves.

The simulation of pollination is thus seen to have presented a complex problem. It is important to keep a clear picture of how it was done, and of its large elements of simplification and guesswork.

\section{(iii) Germination}

One final point arises from a consideration of work on the germination, establishment and reproductive capacity of poppies (Papaver spp.) in natural conditions, summarised by Harper (1966). It was found, as a very broad generalisation, that the number of established plants tended to be constant 
and largely independent of the number of seeds sown. This has some relevance to the situation being considered, because the existence around the interface of hybrids with low fertility and therefore low seed set will mean on average fewer seeds falling per row in that area.

The choice lay between assuming a constant ratio between seeds sown and adults established, and assuming a tendency towards a constant and uniform density of adult plants throughout the experiment. In producing seeds, the model simulated not the total seed output but only the production of successful seed, that is the seed which eventually germinated and produced adult plants. With the first choice, the number of successful seeds produced by a plant would depend only on its fertility. With the second possible assumption, low total seed production in any part of the population, resulting mainly from low fertility, would have to be met by an increased production of successful seeds by all plants in that area. But such an area of the population will have mostly hybrids, since these are the plants responsible for the shortage in total seed production, and to offset low total seed production by increasing the production of successful seed might tend to compensate for the low fertility of the hybrids and so reduce the selection pressure against hybridisation.

This selection pressure was likely to be low, and from an experimental point of view it seemed undesirable to weaken it unnecessarily. Further, Harper's results concerned a weed of agricultural land with well-developed powers of seed dormancy, and there is no reason for believing them to be universally applicable. It was decided, therefore, not to introduce into the basic model any compensation for low total seed production. (In fact, a later experiment showed the effect to be negligible.) Similarly, no account was taken of the effect of plant density on reproductive capacity, which was also demonstrated in poppies in terms of number of capsules produced, and is a well-known phenomenon in agricultural crops and weeds. The reproductive capacities of the model plants were independent of plant density.

\section{(iv) The results of run 16}

In the account which follows, plants of genotypes $16 G$ to $14 G 2 \mathrm{~g}$ are considered as subspecies A, plants of genotypes $2 G 14 g$ to $16 g$ as subspecies $\mathrm{B}$, and plants of genotypes $10 \mathrm{G} 6 \mathrm{~g}$ to $6 \mathrm{GlOg}$ as hybrids (though not all of these will have resulted directly from an act of hybridisation). Plants intermediate between hybrids and subspecies were uncommon, and are ignored in this account; they are of little significance here, although they would be important in an experiment in which introgression was being considered.

The amount of information produced by each analysis of the population was considerable, and such analysis was carried out only at 10-generation intervals. This means that where the term "fluctuation" is used, it refers to fluctuations as seen at 10-generation intervals, and these may not reflect accurately the pattern of fluctuation occurring at single generation intervals.

It would not be possible, except at great length, to give a detailed account of the course of this experiment. Instead, four stages arbitrarily chosen at roughly equal intervals will be used to illustrate the evolutionary progress of the population. To some extent, these may give a misleading impression, especially in so far as they suggest a smooth steady change. It is hoped that 
sufficient care has been taken in the commentary to avoid giving this impression.

The results of each population analysis were output in two ways. Firstly, the population was considered in 128 sections of two adjacent rows, and this allowed the variation in gene frequency through the length of the population to be studied separately for each subspecies and the hybrids. For each tworow section the computer output gave the numbers of $\mathrm{A}, \mathrm{B}$ and hybrids, with for each of these classes the mean numbers per plant of $w$ and $d$ alleles. These results are shown graphically in sections $a, b$ and $c$ respectively of figs. 9-12, which refer to the four exemplary stages of the experiment; except that gene frequencies are not shown for the hybrids, which are merely indicated by position and number at the base of section a of each figure. (The figures are explained in more detail in the caption to fig. 7.)

It should be remembered that for both subspecies at the start the value for $w$ was $2 \cdot 0$ throughout, and that the minimum value of zero corresponds to the minimum possible flowering period of two weeks. For $\mathrm{A}$, the mean starting value of $d$ was $2 \cdot 7$ throughout, and for B it was 3.3 ; a zero value for $d$ corresponded to the earliest possible flowering, and the maximum value of 6.0 to the latest.

The second form of output presented the population in blocks of eight adjacent rows, and for each block it gave for each of the 17 possible $G / g$ genotypes the numbers flowering in each of the eight weeks during which flowering could occur. This gave a fairly detailed picture through the population of the flowering periods of the subspecies and hybrids.

From this information, the histograms of figs. 13-16 have been derived. These consider four 32-row blocks of the population, chosen to be most illustrative so far as was compatible with taking the same four blocks for each of the exemplary stages. These blocks (which were not distributed symmetrically because the population did not remain symmetrical) were the extreme left-hand 32 rows, rows 65-96 which were at about the centre of the zone of interpenetration of all except the first described stage (for which fig. 17 separately shows rows $97-120$ ), rows $121-152$, and the extreme righthand 32 rows. The ranges of these blocks are indicated on fig. $7 \mathrm{a}$.

The histograms show for each block the number of plants of each subspecies (hybrids are omitted) in flower in each week; note that, as with the pilot experiment, it is the number of plants in flower which is given and not the reproductive potential available during each week.

In commenting on the stages chosen to illustrate progress, a number of terms will be used which first require definition.

The zone of interpenetration of the subspecies extends from the leftmost plant of $B$ to the rightmost plant of $A$, inclusively. Hybrids, because of pollen or seed transport may extend outside this zone. Where, in this or any other context, a range is described as extending over a number of rows, this is approximate and could be one more or less.

The potential number of hybrids is the number which might be expected in a particular generation if there were no difference in flowering time between $A$ and $B$ and they were quite free to hybridise. When considered in conjunction with the actual number of hybrids it gives perhaps the clearest indication of progress towards genetic discontinuity of $\mathrm{A}$ and $\mathrm{B}$. It is estimated from the numbers of the two subspecies within the zone of interpenetration in that generation, which is assumed to be not too different 
from the preceding one; the latter of course is the relevant generation, but no population analysis would be available for it. The zone of interpenetration was divided into sections of four rows; if $a$ and $b$ were the numbers of $A$ and $B$ plants in any such section, then the estimated number of potential hybrids for that section was $2 a b /(a+b)$, and this was summed over all sections. It is obvious that this is only a rough estimate, since for example it takes no account of pollen flow between sections or beyond the zone of interpenetration, but one attempted refinement of the calculation made little difference to the estimate, and was clearly not worth the effort.

Gene frequencies for each subspecies separately are expressed as the mean numbers of $w$ and $d$ alleles per plant of that subspecies, either over its whole range or within the zone of interpenetration.

Plant density within the zone of interpenetration is of some interest, since hybridisation, by producing plants of low fertility, should lead to lower seed production and consequent decrease in the number of adult plants in that region. It is expressed as the mean number of plants (of all genotypes) per row over the whole zone of interpenetration; where minimum density within that zone is referred to, this is the mean number per row for that area of 16 consecutive rows for which the plant density is at a minimum.

Generation 10 will occasionally be used to serve as a reference point for the beginning of the experiment, since the actual start had no interpenetration of the subspecies and cannot produce figures for comparison.

In contrast to the pilot experiment, progress in run 16 was very slow. This was to be expected, since the proportion of the total population on which selection could act was small, and gene flow from the rest of the population would have a diluting effect. As was pointed out in an earlier section, we are dealing with a situation in which the rate of progress depends on the extent to which a flowering time difference has developed, and any dilution of this development must slow down progress.

Some interpenetration quickly developed as a result of seed dispersal; at generation 10 it extended over 11 rows which contained 10 plants of $A$ and 31 plants of $B$; there were 25 hybrids.

\section{(a) Generation 140}

By generation 140 (fig. 9), interpenetration had not greatly increased, extending over only 25 rows with 21 plants of $A$ and 81 of $B$. This zone had moved considerably to the left, its centre now being 20 rows left of the centre of the population, with no A plants in B's original half and $166 \mathrm{~B}$ in A's original half. This shift was almost certainly due to the fact that close to the zone of interpenetration and on their respective sides of it $B$ plants had come to occur more densely than A. Thus, in the 20 rows leftwards from this zone there were only $103 \mathrm{~A}$ plants, while in the 20 rows on the other side there were 199 of B. Disparity in these two areas, almost certainly fortuitous in origin, would lead simply by seed dispersal to an excess of B plants within the zone of interpenetration. As pointed out earlier, inequality in numbers in a situation where hybridisation is easily possible produces a positive feedback situation in which the least frequent subspecies finds itself at an increasing disadvantage, because it becomes increasingly more liable to hybridisation. This seems to have happened here, for B plants had become four times as numerous as $\mathrm{A}$ in the zone of interpenetration. Further, not only were there more B plants in a position for invasion, but B invaders would find 
themselves less liable to hybridisation among the sparser $\mathbf{A}$ than would $\mathbf{A}$ invaders among the denser $B$. That is, there was not only more pressure from $B$ to invade (by seed dispersal), but at the same time there was less resistance. $\mathrm{B}$ had advanced, $\mathrm{A}$ had been forced to retreat, and the zone had moved to the left. It would be expected to continue moving until the invading $B$ plants reach an area with a high density of $A$.

There were 13 hybrids in the population, against a potential number of 20. The difference is not significant, and does not itself provide evidence that progress had been made towards supression of interbreeding. Reference to the graphs (figs. 9b and c) and to the histograms (figs. 13 and 17) shows, however, that definite progress had been made. Both $\mathrm{A}$ and $\mathrm{B}$ show a decline in the frequency of $w$ (i.e. a change towards a shorter flowering period), but the shape of the curve for $A$ shows that chance must have played some part there; the decline in $w$ for $\mathrm{B}$ is more consistent, and was clearly greatest in the zone of interpenetration. For the whole subspecies, the $w$ frequencies for $\mathrm{A}$ and $\mathrm{B}$ were 1.64 and 1.43 , and in the interpenetration zone 1.69 and 0.68 respectively.

The change in $d$ frequency (fig. 9c) is more striking, largely because it went opposite ways in $A$ and $B$, but also because several of the farthest penetrating $A$ and $B$ were $6 D$ and $6 d$ respectively, whereas none of the most penetrant $A$ and probably not more than one of the most penetrant $B$ were homozygous with $4 \mathrm{~W}$. In the whole subspecies, the $d$ frequencies for $\mathbf{A}$ and $B$ were 2.82 and 3.62 , while in the zone of interpenetration they were 1.26 and 4.89 respectively. Broadly speaking, the highest values for $d$ in $B$ were in the most invasive plants, as might have been expected; of the seven leftmost $\mathrm{B}$, four were homozygous $d d$ at all three loci, while three were $1 D 5 d$. For these seven plants, the $w$ average was $1 \cdot 0$, corresponding to three weeks of flowering. The three rightmost plants of $\mathrm{A}$ were homozygous $D D$ at all three loci, but two of them were flowering for four weeks and one for five.

There must have been some effect of random change in gene frequency, because $\mathrm{B}$ at the right-hand end of the population had a lower $d$ frequency than it started with, and the $d$ frequency of $\mathbf{A}$ at the left-hand end had also moved against the direction of selection in the subspecies. This can clearly be seen in the histograms a and d of fig. 13, which show that the leftmost plants of A were actually tending to flower later than the rightmost plants of $B$, a reversal of the starting conditions.

However, histograms $b$ and $c$ of the same figure show that on either side of the zone of interpenetration $\mathbf{A}$ had a peak flowering period about three weeks earlier than B, but there was a substantial overlap. Fig. 17 shows separately the flowering histogram for the zone of interpenetration; although there was no week in which both subspecies were not flowering there, most of A's flowering was in weeks 2-4, while most of B's was in weeks 6-8.

As had been expected, the occurrence of substantial hybridisation over a small area had led to a considerable decrease in plant density in the zone of interpenetration (which is reflected in the height of the histogram of fig. 17 , though the fact that this covers only 24 rows instead of 32 must be taken into account), the mean density there being only 4.6 plants per row with a minimum 16-row density of $3 \cdot 0$, as against a population mean of 10 .

It is clear that by generation 140 there were beginnings of a separation in flowering time of the two subspecies, and that a situation had arisen in 
which selection would now be expected to increase this separation more rapidly.

\section{(b) Generations $140-290$}

In fact, though the rate of progress did increase, it did so rather slowly for the next hundred generations, and with substantial fluctuations especially in the extent of interpenetration. The actual movements and numbers of $A$ and $B$ in the zone of interpenetration are of much interest, but require more than a superficial inspection to interpret them. The chance occurrence of one far-flung plant of either subspecies may considerably increase the length of the zone of interpenetration, and as a result substantially affect the number of the other subspecies reckoned to be within the zone. Thus at generation 160 there were $39 \mathrm{~A}$ and $150 \mathrm{~B}$ in this zone, whereas ten generations later the numbers were $38 \mathrm{~A}$ and $34 \mathrm{~B}$; this was largely due to a sharp contraction of $A$ by 14 rows at its right-hand end; these rows, which had contained only four plants of A at generation 160 contained 144 plants of B in generation 170 .

Similarly, a very large increase in the number of $\mathrm{A}$ in the zone of interpenetration in generation 220 was due not to resurgence of $\mathrm{A}$ but to a sudden increase in penetration by B plants of 26 rows, in which there were 260 plants of A. Not surprisingly, the heavily outnumbered B invaders, not yet completely isolated in flowering time, failed to hold their ground, and ten generations later $B$ had retreated by 22 rows. The generations chosen to illustrate the course of this experiment were those least likely to mislead in this way; in any case, the difficulties grew less as the zone of interpenetration increased.

It was not until generation 240 that there was any substantial acceleration in evolutionary progress.

From generation 240, apart from temporary setbacks at 260 and 270 , the extent of interpenetration increased considerably, mainly by leftwards penetration by $B$ in rather large numbers; but there was also some recovery of ground, in smaller numbers, by $A$. The ratio of actual/potential hybrids was also declining rather rapidly, though the actual number of hybrids (spread over many more rows) tended to remain about the same but with large fluctuations. In one short period, at generations 240, 250, 260 and 270, the numbers of hybrids were $4,25,14$ and 6 respectively. 4 was the minimum recorded for the experiment (also at 160, 200, 480 and 490) and 25 was the maximum (also at 10 and 180). (The occurrence of the same number of hybrids in three of the four exemplary generations is pure coincidence.)

\section{(c) Generation 290}

By generation 290 (figs. 10 and 14) interpenetration extended over 85 rows, and the centre of the zone had shifted a further 18 rows to the left. $\mathrm{B}$ plants were again in the majority in this zone, 525 to 320 of $\mathrm{A}$, but on their respective sides of this zone $\mathrm{A}$ and $\mathrm{B}$ were approximately equally dense. The disparity within the zone largely arose from the fact that the extreme rightwards extension of A involved only a few A plants (fig. 10a). There appears to have been little pressure now for further leftward movement of the centre of the zone of interpenetration.

Although there were again 13 hybrids in the population, this is to be compared with the 200 potential hybrids to be expected with the much 

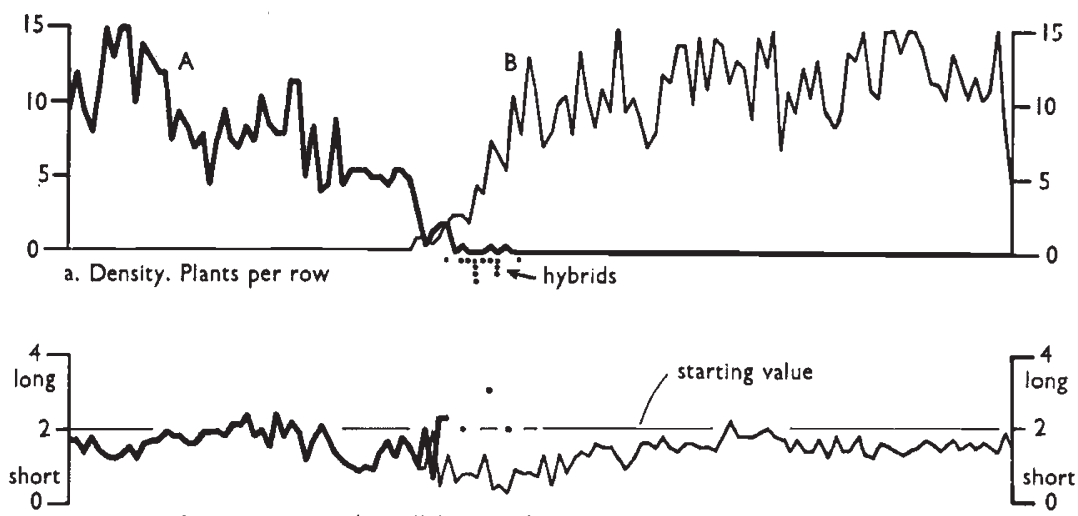

b. Length of flowering period. $w$ alleles per plant

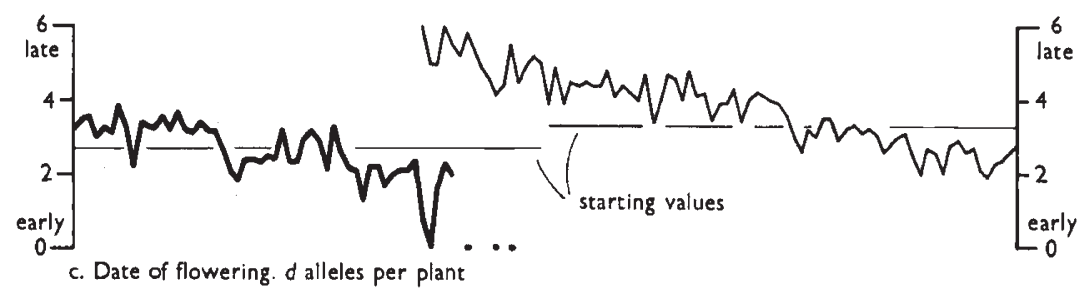

Fig. 9.-The population of run 16 after 140 generations. See legend to fig. 7.
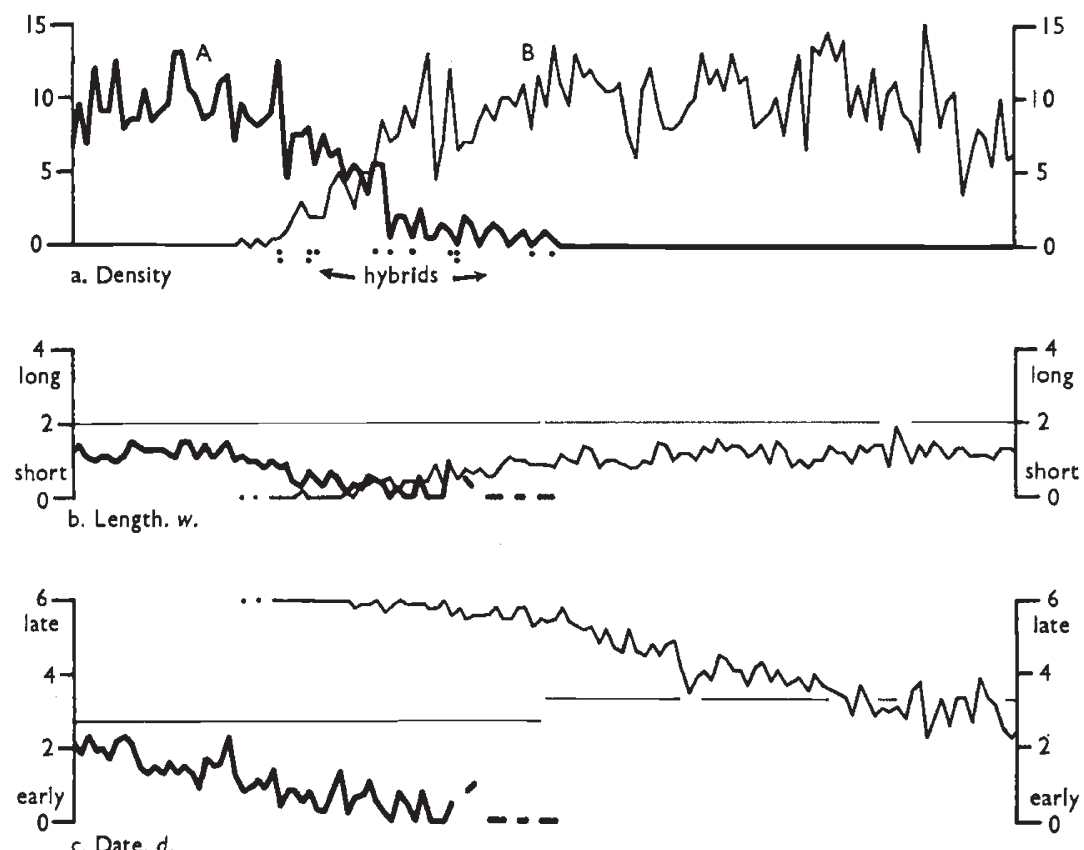

Fig. 10.-The population of run 16 after 290 generations. See legend to fig. 7 . 

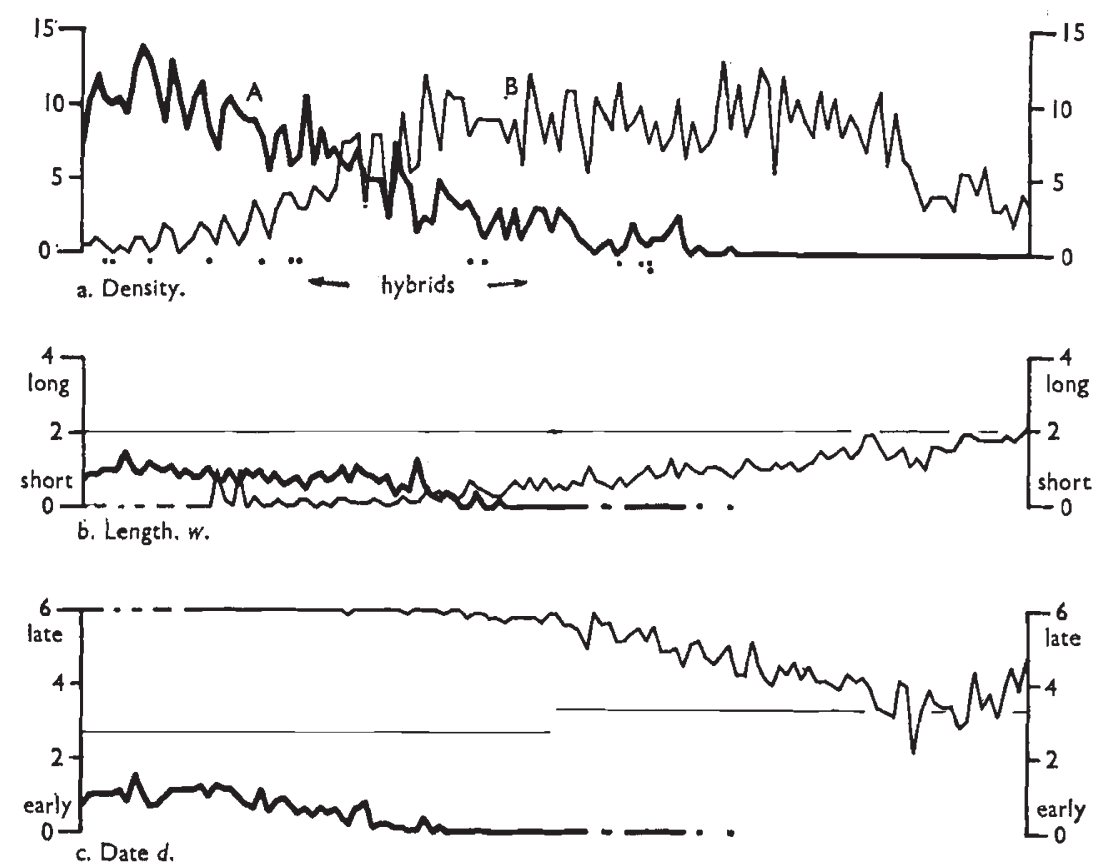

Fig. 11. - The population of run 16 after 430 generations. See legend to fig. 7 .
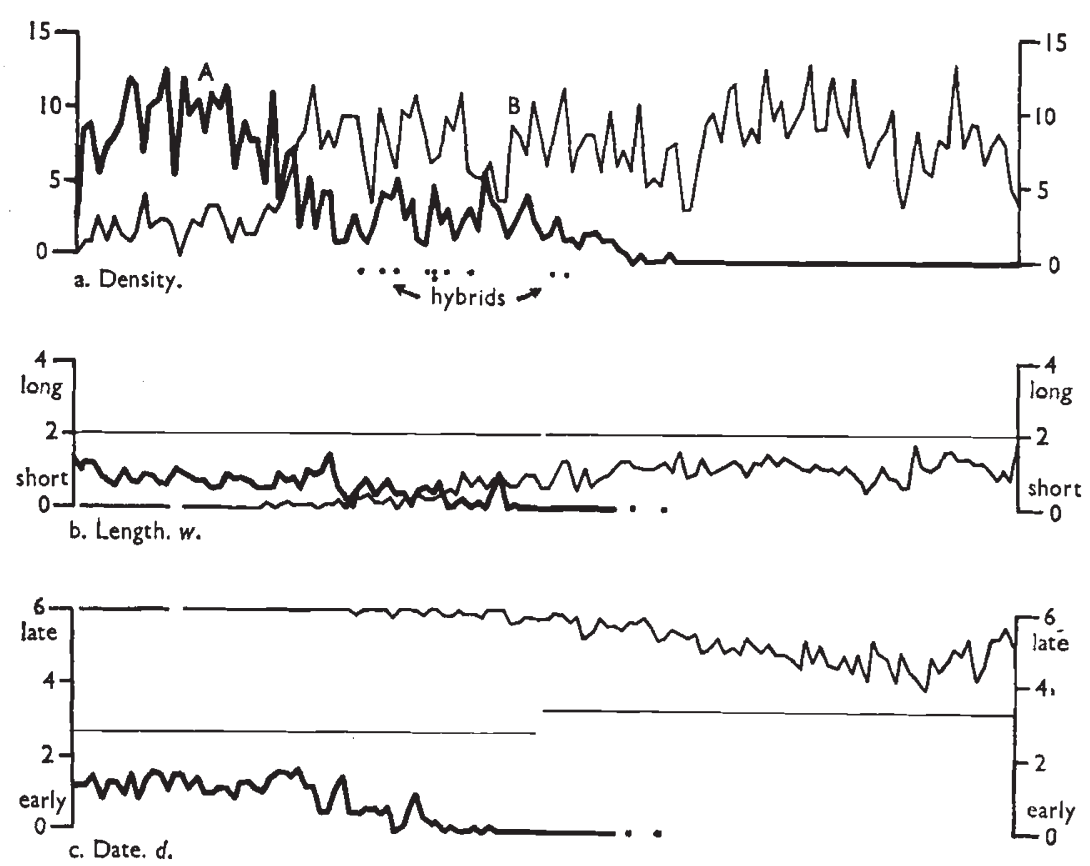

Frg. 12.-The population of run 16 after 540 generations. See legend to fig. 7 . 

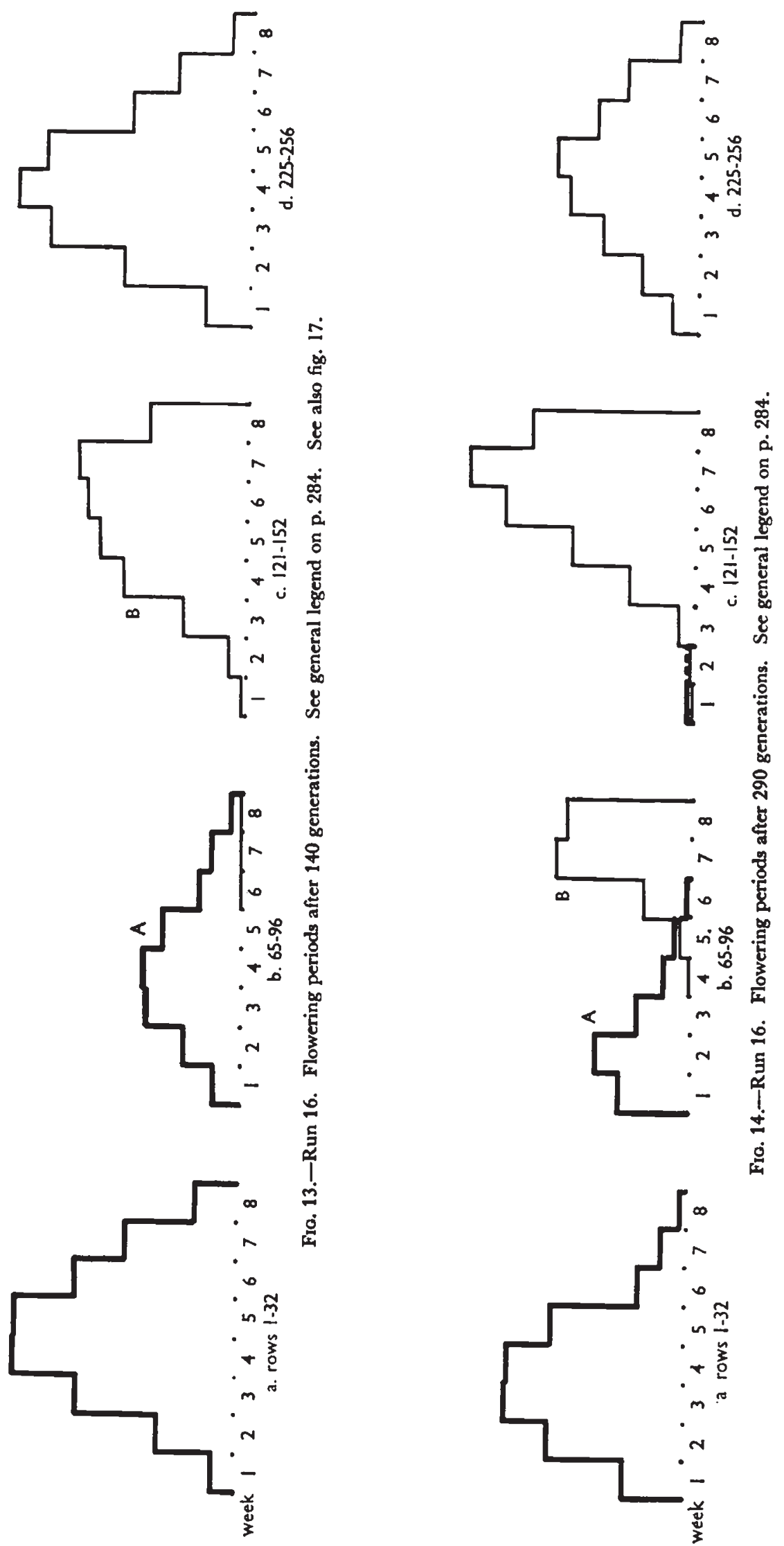

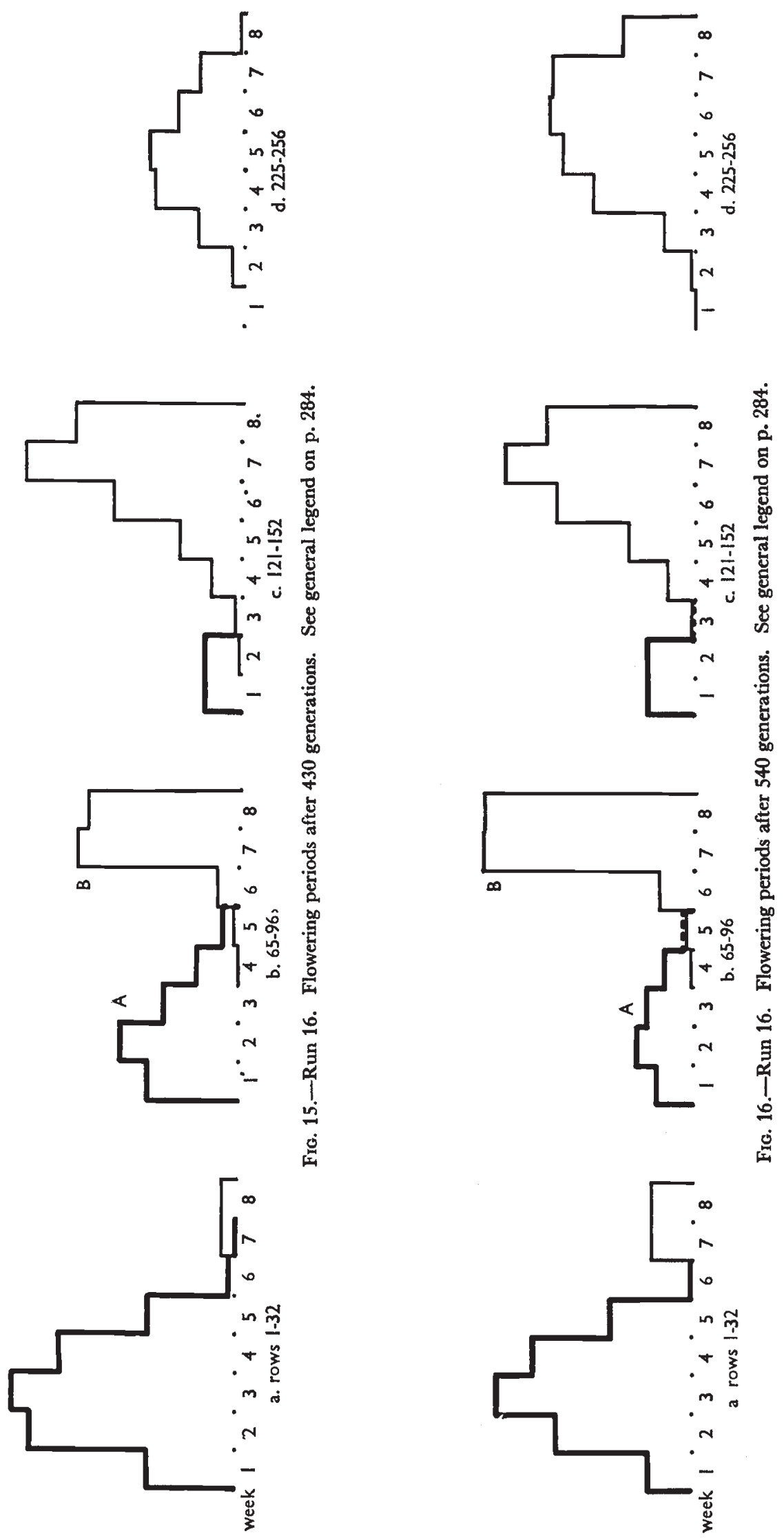


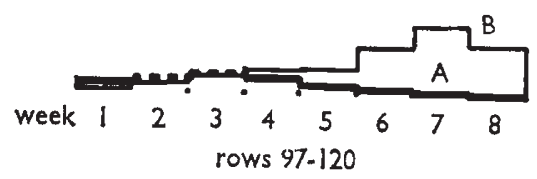

FIG. 17.-Run 16. Flowering periods of the two subspecies after 140 generations in 24 rows of the zone of interpenetration, as indicated at the top of fig. 7a. For each week, the number of plants of each subspecies in flower is shown. See also fig. 13. The scale is the same as in figs. 13-16.

increased numbers of both subspecies occurring intermingled. Why this number was not even approached is clear from fig. $10 \mathrm{~b}$ and $\mathrm{c}$ and from the flowering histogram of fig. 14b which covers rows 65-96, where A and B are roughly equally abundant. Here, there was relatively little simultaneous flowering of A and B, and that was confined almost entirely to week 5 . The possibility of hybridisation had obviously decreased considerably, and this was reflected in the fact that there was no longer any shortage of plants in the zone of interpenetration, the mean density there being 10.0 plants per row, the mean for the population, with a 16-row minimum of 8.9.

There had clearly been a sharp evolutionary change in the floweringtime situation, and the considerable gene frequency changes in the zone of interpenetration (fig. $10 \mathrm{~b}$ and c) were beginning to spread outwards to the remainder of each subspecies. Among the interpenetrating A, $w$ frequency had fallen from 1.69 at generation 140 to 0.56 , and for the whole subspecies from 1.64 to 0.97 . Among the interpenetrating $B, w$ frequency had changed little, from 0.68 to 0.62 , but for the whole subspecies it had fallen from 1.43 to 1.01 . Change in $d$ frequency was also striking; for interpenetrating $\mathbf{A}$ it had fallen from 1.26 to 0.69 , and for all A from $2 \cdot 82$ to 1.27 ; for interpenetrating $B$ it had risen from 4.89 to 5.73 (which is near the maximum) and for all B from 3.62 to 4.48 .

The effect for both sets of genes was again most marked in the most penetrant plants of each subspecies. The 14 rightmost $A$ were all confined in flowering to the first two weeks, and 52 of the 54 leftmost B were confined in flowering to the last two weeks. The selective advantage of evading hybridisation is greatest for a subspecies where it is most heavily outnumbered.

The occurrence of centrifugal flow of the selectively favoured alleles is suggested both by the gradients of fig. $10 \mathrm{~b}$ and $\mathrm{c}$, and also by a comparison of fig. $14 \mathrm{a}$ and $\mathrm{d}$ with fig. $13 \mathrm{a}$ and $\mathrm{d}$. In the leftmost 32 rows of the population, the peak flowering of $A$ at generation 290 was a week earlier than it had been at 140, and the peak flowering of $B$ in the rightmost 32 rows had become later by a week. The previous tendency for the $d$ frequencies at the ends of the population to move against the direction of selection had been reversed. To some extent this reversal, like the original movement, may have been fortuitous; but looking at the graphs it is difficult to believe that centrifugal flow of the selected alleles had not been of some importance.

Fics. 13-16.-Run 16. The flowering periods of the two subspecies (as defined in fig. 7) in four 32-row sections, showing for each section the numbers of plants of each subspecies in flower in each week. The vertical scale is the same throughout; the peak in fig. 13d represents 302 plants. The positions of the sections, which are the same for all four figures, are indicated at the top of fig. 7a. Where the lines for A and B coincide, a line of alternating thickness is used. 


\section{(d) Progress of interpenetration after generation 290}

From generation 290, very rapid progress in interpenetration was made for about 30 generations, during which period the zone of interpenetration almost doubled in size. At generation 330, there was a B plant in the leftmost two rows of the population, but this advance was only temporary and ten generations later there was only one B in the leftmost 30 rows, while at 410 there were no $B$ at all in the leftmost 40 rows. In fact, from generation 320 there was a great deal of fluctuation in the extent of interpenetration, both in terms of rows and of numbers of plants of $A$ and $B$, and it would be difficult to justify a belief that there was any permanent increase in interpenetration from generation 320 to the end of the experiment at 540. A's most successful time was at generation 410 when there were $57 \mathrm{~A}$ plants to the right of the centre of the population (i.e. in an area originally occupied by $B$ alone); all of these plants were confined in flowering to the first two weeks, during which period in the same area there were practically no B plants in flower. By generation 530, the number of A plants right of the population centre had fallen to five, but when the experiment was stopped ten generations later it had risen suddenly to 38 .

B's most successful periods were at generations 460 and 540. At 460 there were $52 \mathrm{~B}$ plants in the leftmost 32 rows, all confined in flowering to the last two weeks, during which period in the same area none of the $313 \mathrm{~A}$ plants were flowering; this local reproductive isolation was maintained except for occasional A plants flowering in week 7. There was some later falling off in density of $B$ at the left-hand end, but at the end of the experiment there were $53 \mathrm{~B}$ plants in the leftmost 32 rows.

Changes in gene frequency pursued a more regular course, continuing the progress which has already been described up to generation 290 .

\section{(e) Generation 430}

Fig. $11 \mathrm{~b}$ and c shows the graphs of $w$ and $d$ frequencies at generation 430 , and requires little comment except to point out that for each subspecies the most penetrant plants (in considerable numbers) were almost entirely homozygous both for $W$ and for $D(\mathrm{~A})$ or $d(\mathrm{~B})$. Indeed, to the right of row 100 all A plants (110 in number) were homozygous $6 D$, and to the left of that row 321 out of $323 \mathrm{~B}$ plants were homozygous $6 d$.

In spite of this, the changes in mean gene frequency (compared with generation 290) within the zone of interpenetration were relatively slight, but this was largely due to substantial extension of the zone, with considerable differences between the most and least penetrant plants of each subspecies. For the whole subspecies, however, the mean gene frequencies had changed considerably. For A (at this generation entirely within the zone of interpenetration) the mean value of $w$ had fallen from 0.97 to 0.77 , and $d$ from 1.27 to 0.70 . For $\mathrm{B}$, the mean value of $w$ had fallen from 1.01 to 0.83 , and that of $d$ had risen from $4 \cdot 48$ to $5 \cdot 07$.

The consequences of the changes in gene frequency can be seen from the histograms of fig. 15. Where A and B occurred most abundantly together (15b), there was very little overlap in flowering time, A mostly flowering in weeks $1-4$ and $B$ in weeks 7 and 8 . Histograms a and c show that there was even less overlap in flowering at the ends of the zone of interpenetration, where the invading subspecies was restricted to the first or last two weeks and the 
invaded one flowered over a rather broader period than it did in the centre of the zone.

The continued shift of $\mathbf{B}$ at its right-hand end towards later flowering (fig. 15d) again supports the idea of centrifugal gene flow, and this could be deduced from fig. 11c.

Although there were now many more A and B plants co-existing, it is clear that the possibility of hybridisation had decreased considerably. While the number of hybrids in the population was again 13, the potential number was now 526, as compared with 200 at generation 290 . Only 2.5 per cent. of the potentiality for hybridisation had been realised.

By generation 430 the two subspecies had, by selection of genes controlling date and length of flowering, achieved a high degree of reproductive isolation.

\section{(f) Generation 540 (end of experiment)}

In the following 110 generations to the end of the experiment at 540 (figs. 12 and 16), there was relatively little increase in the extent of reproductive isolation, except that the $d$ frequency in B continued to rise at the right-hand end of the population, where the peak flowering period was now about two weeks later than it had been at 430 . There were now 10 hybrids in the population, compared with a potential number of 557 . It is worth noting that they were mostly towards the centre of the zone of interpenetration, and not around its limits. This tendency had been developing fairly steadily, and was clearly correlated with the much more pronounced separation in flowering time of A and B at the limits of the zone of interpenetration than at its centre, where there still remained a little simultaneous flowering. Indeed, at each end of the zone of interpenetration there was a week in which hardly any plants of either subspecies were flowering. In the area covered by fig. 16a there were only two plants (both $\mathrm{A}$ ) flowering in week 6 ; in the area covered by fig. $16 \mathrm{c}$ there were only three plants flowering in week 3 -one of these was $A$ and was separated by at least eight rows from the other two, which were $B$.

Further changes in gene frequency, with one exception, had been slight and may well have been due to chance, especially as some of them were against the direction of selection. The frequency of $d$ in $\mathrm{B}$ at the right-hand end of the population had continued to rise, and was now well above its starting value; it will be remembered that at generation 140 it had been well below its starting value. This increase is reflected in the $d$ frequency for the whole subspecies, which had risen since generation 430 from 5.07 to $5 \cdot 39$, the comparable increase for $\mathbf{B}$ within the zone of interpenetration being from $5 \cdot 65$ to $5 \cdot 86$.

\section{(g) Discussion of results of run 16}

This experiment demonstrated quite clearly that in the model population selection against the low fertility caused by hybridisation could bring about an almost complete separation in flowering time, both by shortening the flowering period and by advancing or retarding the date of flowering, and could allow the two subspecies to intermingle freely with little hybridisation and gene flow between them. Before the end of the experiment, A and B had effectively become two distinct species. Over the 540 generations the mean frequency per plant of $w$ had fallen from 2.0 to 0.64 in $\mathrm{A}$ and 0.81 in 
B; the mean frequency of $d$ had fallen in A from 2.7 to 0.98 and had risen in B from 3.3 to 5.39 . The results are summarised in table 2.

Effectively, this may well have been the end of the process, and there are no grounds for believing that had the experiment been continued after generation 540 there would have been any substantial change within a reasonable time. Hybridisation no longer had any appreciable relevance to the question of interpenetration; it seems likely that diffusion resulting from seed dispersal would slowly cause each species to become more evenly distributed through the entire length of the population, but this would

TABle 2

Summary of evolutionary progress in run 16

"frequency" means number of the specified alleles per plant.

"z. ip." means zone of interpenetration.

"ppn." means whole population.

Generation

No. of rows in z. ip.

No. of $A$ in z. ip.

No. of $B$ in z. ip.

No. of hybrids

Potential no. of hybrids

Frequency of $d$ in $\mathrm{A}$ in z. ip.

Frequency of $d$ in $\mathrm{A}$ in ppn.

Frequency of $w$ in $\mathbf{A}$ in z. ip.

Frequency of $w$ in $\mathrm{A}$ in ppn.

Frequency of $d$ in B in z. ip.

Frequency of $d$ in $B$ in ppn.

Frequency of $w$ in $B$ in $z$. ip.

Frequency of $w$ in $\mathrm{B}$ in ppn.

Minimum 16-row density in z. ip., as plants per row

$\begin{array}{rrrrr}10 & 140 & 290 & 430 & 540 \\ 11 & 25 & 85 & 175 & 159 \\ 10 & 21 & 320 & 904 & 752 \\ 31 & 81 & 525 & 1021 & 927 \\ 25 & 13 & 13 & 13 & 10 \\ 8 & 20 & 200 & 526 & 557 \\ 2.86 & 1.26 & 0.69 & 0.70 & 0.97 \\ 2.51 & 2.82 & 1.27 & 0.70^{*} & 0.98^{*} \\ 2.42 & 1.69 & 0.56 & 0.77 & 0.63 \\ 1.81 & 1.64 & 0.97 & 0.77^{*} & 0.64^{*} \\ 3.56 & 4.89 & 5.73 & 5.65 & 5.86 \\ 3.39 & 3.62 & 4.48 & 5.07 & 5.39 \\ 2.47 & 0.68 & 0.62 & 0.53 & 0.50 \\ 2.07 & 1.43 & 1.01 & 0.83 & 0.81 \\ & & & & \\ - & 3.0 & 8.9 & 8.9 & 9.5\end{array}$

* At 430 and 540, all or almost all A were within the z. ip.

certainly have taken a very long time, and would have involved the use of much more computer time than could have been justified by the amount of information likely to be obtained.

With the genetical structure used in this model, hybridisation would probably never be eliminated completely, largely because more genetical variability was available than required to ensure flowering-time separation of $\mathrm{A}$ and $\mathrm{B}$. This point has been discussed in relation to the pilot experiment, and may be expanded here.

Reference to fig. 12 shows that at the left-hand end of the experiment, B was genetically fixed at $4 \mathrm{~W}$ and $6 d$ and confined in flowering to the last two weeks, whereas $\mathrm{A}$ had considerable genetic variability and was flowering almost entirely during the first five weeks. At the right-hand end of the zone of interpenetration it was $\mathrm{A}$ that was almost completely fixed, at $4 \mathrm{~W}$ and $6 D$, and $\mathrm{B}$ which was genetically variable and flowered almost entirely during the last five weeks. There is no discontinuity in gene frequency within each species, but a fairly steady gradient; so there must be a region around 
the centre of the zone of interpenetration where both species show genetic variability. It is inevitable that this variability should occasionally produce plants part at least of whose flowering time would be within the range of flowering of the other species; hybridisation would then still be liable to occur. Since selection depends on the occurrence of hybridisation, as the latter becomes rarer so the divergence of flowering time as a result of selection becomes slower, and might never be complete. It could however become complete as a result of genetic drift, though not necessarily permanently.

If the density of each species through the population levels out by diffusion, it would be expected that gene flow within each species would cause the gradients in gene frequency to level out, and the genetic fixation at the rightmost end of $A$ and the leftmost end of $B$ would disappear, each species becoming variable through its length. This is a different situation from the one seen at the end of the pilot experiment, but it is conceivable that random genetic drift could bring about genetic fixation in one species, with a flowering period confined to the last or the first two weeks.

But it is obvious that in this model at least all of the possibilities which have been discussed would take a very long time indeed. It seems reasonable to suppose that they would also take a very long time in nature, and the population constitution at the end of the experiment may well resemble the reality of many natural situations. If that were the case then where two closely related taxa, able to hybridise, have areas of distribution which partly coincide, it might be expected that at the boundaries of their common area the taxon which was at its limit of distribution would have the shorter and less variable flowering period, if that were the principal isolating factor.

It was pointed out earlier that in Britain Primula veris has a shorter flowering period than $P$. vulgaris; it is $P$. veris which reaches the westerly limits of its range as a common species before vulgaris, which extends abundantly further westwards. On the continent of Europe, $P$. veris extends further north and east than vulgaris, and it would be interesting to know whether the relative lengths of the flowering periods of these two species are reversed in west- and south-central Europe and in Scandinavia.

One considerable difference between the pilot experiment and run 16 concerned the purity of the subspecies. It will be remembered that in the pilot experiment the pure subspecies, $16 G$ and $16 g$, had almost disappeared from the population by generation 32. Although these genotypes increased in numbers later, there were still about 25 per cent. of plants which were neither $16 G$ nor $16 g$ at generation 80 , when flowering time isolation was almost complete. Obviously, in run 16 plants well away from the zone of interpenetration are likely to be pure A or B; but even within that zone there was always a large majority of $16 G$ and $16 \mathrm{~g}$ plants, so that the subspecies nowhere lost their identity, as they had in the pilot experiment. At generation $140,99 \cdot 1$ per cent. of $A$ were $16 G$ and 98.8 per cent. of B were $16 g$. At the end of the experiment, the figures were 93.7 and 99.7 respectively.

\section{(v) Centrifugal gene flow}

Although run 16 suggested that there was flow of favoured alleles outwards from the zone of interpenetration, with change in flowering period extending to plants of subspecies B well away from contact with A, it was not an entirely convincing demonstration. This was partly due to the 
considerable size of the zone of interpenetration at the end of the experiment, when only part of B and none of A extended outside it (fig. 12).

An experiment was carried out with a modified model which restricted the size of the zone of interpenetration in a natural way, but which was otherwise identical with the model of run 16. The restriction was carried out by supposing that $\mathrm{A}$ and $\mathrm{B}$ had different ecological preferences. Rows 1-112, initially occupied only by $A$, were more favourable for A plants; these had the same reproductive capacity there as in run 16, whereas $\mathbf{B}$ plants in those rows had only three-quarters of their reproductive capacity in that run. In rows $145-256$ the situation was reversed, with $B$ the favoured subspecies. The central 32 rows were neutral territory, both $A$ and $B$ having the full reproductive capacity as in run 16 . Hybrids had no ecological preference, and wherever they occurred had the same reproductive capacities as in run 16. The consequence of this was that either subspecies invading the territory of the other was at an additional disadvantage, and the ecological difference was sufficient to restrict the zone of interpenetration to little more than the central 32 rows, throughout the entire experiment.

This experiment will not be described in detail, but the end-point at generation 510 is illustrated by figs. 18 and 19. The result was striking, and provides a clear demonstration of centrifugal flow of the alleles selected in the centre of the population. Comparison of fig. 19a and $c$ shows that the leftmost A plants had little overlap in flowering time with the rightmost B plants, although each was well separated from the nearest plants of the other species. Obviously, the scale of the model is not nearly large enough to provide a complete refutation of Moore's (1957) opinion, referred to previously, but at least it demonstrates that development of differentiation in flowering time need not be confined to the region where the two subspecies are in contact.

The greater progress made by this experiment towards flowering-time separation than was made in run 16 was probably largely a matter of chance. Early generations in these experiments, when a selective situation is developing, are particularly affected by random fluctuations, and on this occasion the evolutionary stage reached by the population by generation 120 roughly corresponded to that reached in run 16 by generation 260 . It will be remembered that in the rightmost plants of B in run 16 there was initially a considerable random drift in $d$ frequency against the direction of selection.

\section{(vi) Further experiments}

Once a sensible model of this kind has been established, it is easy to modify it in many ways so that a whole series of experiments may be performed. Some of the more interesting results from such experiments will be described briefly.

\section{(a) Control-no loss of fertility in hybrids}

A control run was carried out in which there was no loss of fertility in the hybrids, all genotypes having identical fertility. As would be expected with the hybrids able to reproduce freely, there was rapid mixing of $G$ and $g$ alleles through hybridisation and backcrossing of hybrids to the subspecies. Pure A and B disappeared from the centre of the population, and intermediate genotypes increased there in number and range. The situation after 140 generations bore little resemblance to run 16 at the same stage. T 2 
There were 471 " hybrids" (i.e. $10 G 6 g-6 G 10 g$ ) though few of these were hybrids in the strict sense of originating from crossing between $\mathbf{A}$ and $\mathbf{B}$; this was hardly possible any longer, since there was only one plant of $A$ (which was $14 G 2 \mathrm{~g}$ ) and one of $\mathrm{B}(2 \mathrm{Gl} / \mathrm{g})$ in the central 28 rows of the population. There was only one pure $\mathrm{A}(16 G)$ and one pure $\mathrm{B}(16 \mathrm{~g})$ in the central 80 rows. The " hybrids", which extended over the central 100 rows,
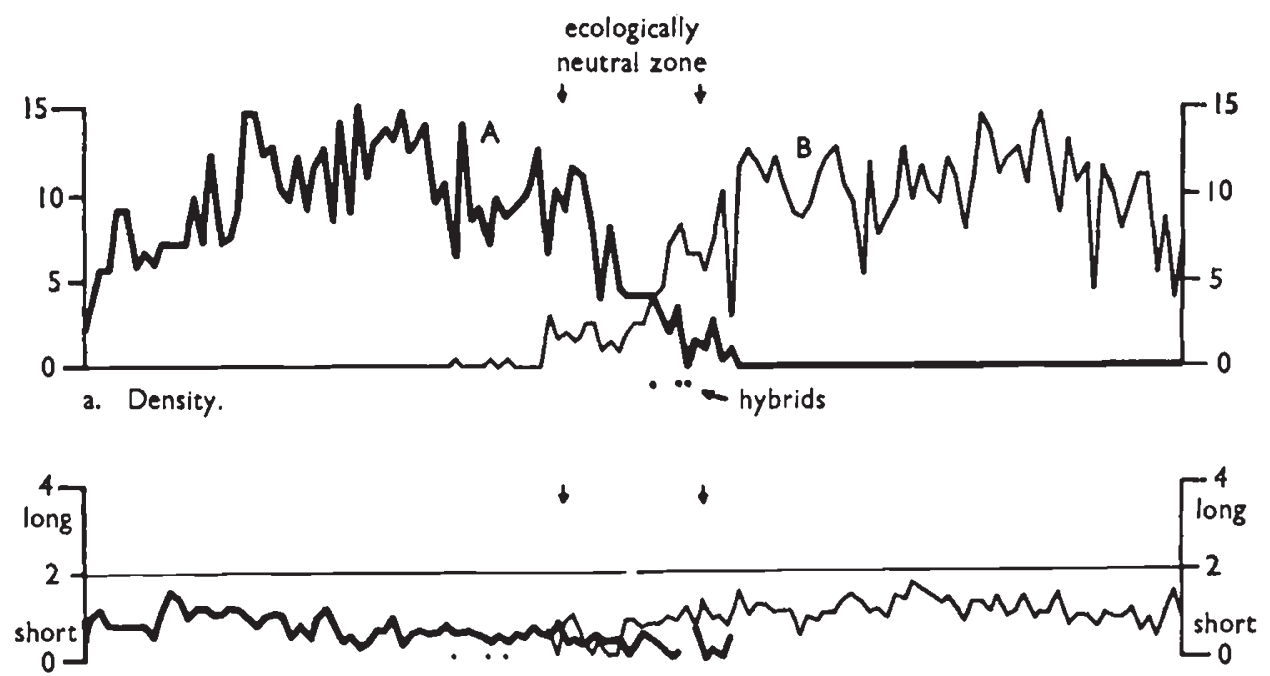

b. Length. w.

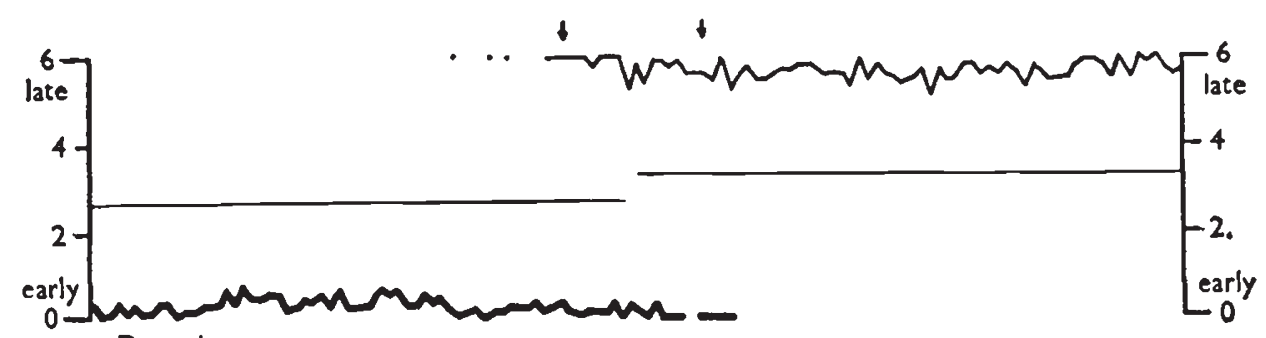

c. Date. d.

Fio. 18.-The population of the run with ecological differentiation, after 510 generations. See legend to fig. 7 . The arrows indicate the limits of the ecologically neutral zone.

must have been the offspring of plants of similar genotypes. Of plants intermediate between hybrids and subspecies, which occurred insignificantly in run 16, there were now 668 occurring throughout the length of the population. There was no drastic drop in plant density in the centre as there had been in run 16; in fact, the mean density there was somewhat above the population average.

The overall frequencies of $w$ and $d$ fell during the control experiment. It is difficult to be certain, but it seems very likely that these falls were due to random fluctuation. There were considerable fluctuations in plant density throughout the population, as well as in gene frequency. The latter seems to have been most pronounced when and where plant density showed drastic but temporary reduction. The gene frequency distributions through the population, especially those of $d$, bore little resemblance to those at 


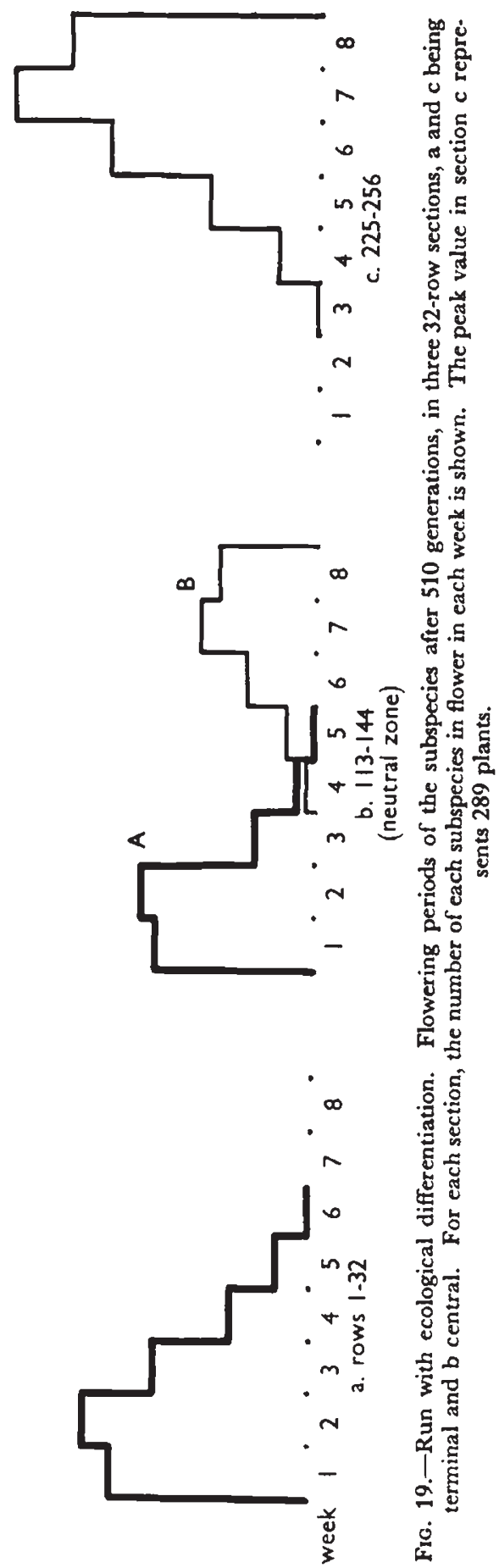


generation 140 of run 16, and had little relevance to the distribution of the subspecies and the hybrids, the pattern being quite devoid of symmetry.

The overall frequency of $w$ fell to about the same value as it had reached in run 16 , but it did so unevenly through the population; in the leftmost 50 rows it did not fall at all. Its minimum value was about 30 rows to the right of the centre of the population and was distinctly higher than the minimum value for run 16 at 140 generations, which was in the zone of interpenetration. It is clear that random fluctuation cannot be ruled out as being at least partly responsible for the fall in frequency of $w$ in run 16; but the clear relevance of the distribution pattern of $w$ frequency to the zone of interpenetration throughout run 16, when contrasted with the lack of such relevance in the control run, and especially the fact that in run 16 the most penetrant plants of each subspecies eventually reached minimum possible values, makes it clear that there must have been some effective selection against $w$ in run 16.

For $d$ frequency at generation 140 , control and run 16 were clearly different. In the control, $d$ frequency in $A$ had risen from 2.7 to 3.0 and was fairly uniform throughout $A$; in $B$, the mean frequency had fallen from 3.3 to $3 \cdot 1$, but there was a steep gradient falling from about 3.9 at the extreme right of the population to about 1.4 at the left-hand end of $B$, where the hybrids showed a similar value. The clear difference between the patterns of $d$ frequency distribution through $\mathbf{A}$ and $\mathbf{B}$, where the genetical situations are essentially identical, makes it very probable that the fall in $d$ frequency, occurring mainly in the 32 rows right of the population centre, was fortuitous. It took place principally in the first 90 generations, when it fell to a mean value of 1.57 over those 32 rows. It subsequently fluctuated there with a minimum value of $1 \cdot 29$, but had risen to 1.56 again by generation 170 , when the run was discontinued.

The control showed nothing resembling the opposite changes in $d$ frequency in A and B shown by run 16 at generation 140, when the most penetrant plants of $\mathrm{A}$ had the minimum possible value for $d$ and those of $\mathrm{B}$ had the maximum. The control run gave good support for the view that the changes in date of flowering in run 16 were the result of selection for the avoidance of hybridisation.

\section{(b) Interpenetration of $A$ and $B$ at start}

In a naive attempt to accelerate the selection process at the start by increasing the possibility of hybridisation, one experimental run was carried out with the two subspecies at the beginning interpenetrating uniformly over the central 32 rows (five plants of each per row), instead of being simply in contact. This actually produced the opposite of the desired effect, which is hardly surprising (see discussion near beginning of section 3 ). During the first ten generations the extent of interpenetration decreased to seven rows, plant density in the central 32 rows to 3.4 plants per row, and there was only one hybrid in the tenth generation. The correspanding figures for run 16 were 11 rows, 4.7 plants per row and 25 hybrids.

\section{(c) Reproductive advantage in longer flowering}

The necessity for assuming (in the basic model) that total reproductive capacity of a plant was independent of the length of the flowering period 
was tested in an experiment in which a small advantage was given to plants which flowered for longer times. If the total reproductive capacity of a plant flowering for two weeks be taken as 1 , then 0.041 was added to this for each week a plant flowered in excess of two. Otherwise, the model was exactly as in run 16 . The selective advantage of longer flowering was relatively small, but was quite enough to swamp the second-order selective advantage in the opposite direction. There was a rapid increase (from the initial $2 \cdot 0$ ) in mean frequency of $w$ (towards longer flowering) in both subspecies, reaching a value of about 3.0 by generation $30,3.5$ by 90 , and 3.87 by 150 when almost 90 per cent. of the population were $4 w$ and flowering for the maximum period of six weeks. The frequency of $w$ was no lower in the centre of the population than elsewhere.

The run was not continued after generation 150 . Then, the subspecies interpenetrated over only 13 rows; $d$ frequency of $A$ in the zone of interpenetration had fallen only to 1.6 and of $\mathrm{B}$ had fallen slightly to 3.0 (comparable values at generation 140 of run 16 were 1.26 and 4.89 respectively).

It seems clear that a small reproductive advantage in longer flowering was sufficient to reduce drastically the rate of selection for difference in flowering time. With almost all plants flowering for six weeks, there could not be less than four weeks during which the subspecies flower simultaneously and this must reduce any effectiveness of lateness or earliness in flowering and the strength of any selection at the $D / d$ loci. The results of this run emphasise the weakness of the second-order selection pressures involved in the avoidance of hybridisation.

\section{(d) Germination and seed density}

The basic model assumed that the proportion of seed which germinated and grew to maturity was independent of seed density on the ground. An experiment was performed in which germination in areas of low seed production was increased (by producing additional germinable seed there), so that a minimum of eight plants per row was maintained. Since low seed production occurs primarily in areas where there is a majority of hybrids, this has the effect of increasing the reproductive capacity of hybrids and so reducing the disadvantage of hybridisation. But once some degree of flowering-time isolation has developed there will be fewer hybrids and these will be more dispersed and so have little effect on seed density. The model would then become essentially the same as in run 16. In fact, it made only a slightly slower start than run 16 (and this might well have been chance), and by generation 60 there was little difference between the two. Whatever effect compensation for low density may have been having, it was certainly slight by then; after generation 60 , and presumably as a matter of chance, selection for flowering-time differentiation proceeded more rapidly than it had in run 16. By generation 200 , the position was essentially the same as that at generation 540 of run 16, except that this time it was $\mathrm{A}$ which extended over the whole population and B which occurred through only about half of it. From generation 220 to 360 , there were on average only 3.3 hybrids per generation, and generations 340,350 and 360 (the last of the run) had 0,0 and 1 hybrid respectively. This run showed that compensation for low seed density had little effect; at the same time, it provided a clear confirmation of the validity of the results of run 16 . 
(e) Minimum fertility not with $F_{1}$ hybrids

It sometimes happens that while $F_{1}$ hybrids are fairly vigorous, $F_{2}$ segregates and first backcrosses are considerably weaker; this has been attributed to what is often called, rather vaguely, gene unbalance. If loss of reproductive capacity is due to loss of vigour (in terms of flower production) rather than to gamete sterility, then minimum reproductive capacity will not now be with the $\mathrm{F}_{1}$. An experiment was performed in which $12 \mathrm{G} 4 \mathrm{~g}$ and $4 G 12 \mathrm{~g}$ plants had the minimum fertility of 10 per cent. (pure subspecies having 100 per cent.), while $8 G 8 g$ plants had a fertility of 75 per cent. Flowering-time differentiation developed more rapidly than in run 16 and with increasing momentum; this was due at least partly to the fact that the mean fertility exclusive of $\mathrm{A}$ and $\mathrm{B}$ was somewhat lower than in run 16. There was a substantial change between generations 40 and 60 , and by generation 140 the subspecies were interpenetrating over 63 rows; there were four central hybrids and six plants between these and A or B. It was noticeable throughout the run that a higher proportion of $\mathrm{A}$ and $\mathrm{B}$ were pure $16 G$ or $16 g$ than in run 16 .

\section{(f) Plants treated as perennials}

All the experiments so far described treated the plants as annuals. An experiment was carried out in which the plants were perennials with a mean life-span of 7.5 years, flowering equally well in all years. One of the consequences of perennation is an increase in the effect of random fluctuation (Crosby, 1966b), since the genetic variance of a perennial population is roughly the same as that of an annual population of about half the size. It was now clear, as had been suspected for some time, that the random fluctuations in the original model population were close to the limit of tolerability, for this perennial experiment was largely dominated by random fluctuations. By generation 20 there was clear divergence between A (early) and $B$ (late) in the zone of interpenetration which covered 33 rows, but this was less clear 20 generations later, when the three rightmost plants of $\mathrm{A}$ were $1 D 5 d, 6 D$ and $6 D$ and were flowering for 4,6 and 6 weeks respectively. There was much subsequent fluctuation in gene frequency in the zone of interpenetration, and by generation 120 both subspecies were tending to flower early. In generation 130, A was flowering later than B in the 27-row zone of interpenetration, the $d$ frequencies being 2.46 and 1.20 respectively; the 14 most penetrant A plants actually averaged 4·16.

The situation was reversed again at generation 200 , but both subspecies were tending to flower early. Interpenetration was then over 17 rows, and there were 15 hybrids. The $d$ frequencies there were 1.42 for $\mathrm{A}$ and 2.08 for $B$, and the mean lengths of flowering were 3.3 and 3.8 weeks. Obviously, little permanent progress was being made because random fluctuation was swamping selection; it seemed pointless to continue the experiment, which might have gone on in this way for a long time before chance so tipped the balance that selection could have become strong enough to take over.

\section{(g) Change in breeding system}

A whole series of experiments was performed with a different basis for genetic isolation, namely a change from outbreeding to self-fertilisation. A self-fertilised plant cannot itself produce hybrid seed, but its pollen might 
still hybridise the ovules of a related subspecies which remains outbreeding. This situation has been discussed by Crosby (1966a) and is a very complex one to deal with. One hardly begins to realise the full complexities until one tries to construct a computer model. For instance, a critical factor which has to be properly balanced in the model is inbreeding depression; in nature this would not be expected to remain constant during evolution towards inbreeding, and this ought to be taken into account in a model.

Several successful experiments were carried out with the breedingsystem model, but they posed more genetical questions than they solved. They will not be described here, since pressure of other duties compelled me to put the problem on one side. It is hoped to take it up again using larger populations on a faster machine.

\section{Discussion}

There is little need for detailed discussion of the results described in this paper. They have demonstrated clearly that, although small, selection pressure against hybridisation, when this produces hybrids of low fertility, is sufficient to lead to the evolution of pre-fertilisation genetic barriers to interbreeding and so to the production of genetic discontinuity which is the fundamental element in the process of speciation. They provide support for the view that the allelic differences involved in the isolating mechanism need not be confined to the region of contact of the taxa concerned, which is where selection occurs, but the alleles concerned may spread and so change in frequency away from it.

The simulation of position and distance is a valuable technique which is easily capable of greater development, and could well be used for example in the study of situations involving considerable ecological complexity.

The problem of the evolution of genetic discontinuity could hardly have been tackled by any other method. One important advantage which this kind of computer simulation method has over mathematical models is that it allows populations to be considered in terms of the individual plants which compose them. There were many occasions when the ability to consider the genotypes of specific individual plants enhanced the understanding of the evolutionary processes.

The main weakness of this work was that the population size was barely large enough, and allowed perhaps too much random fluctuation. The actual number of plants in the evolutionarily active region (in and adjacent to the zone of interpenetration) was quite small in the early stages of each experiment. Substantially increasing the proportion of the population within this region (by increasing the range of pollen and seed dispersal) would have mitigated this disadvantage, but would have tended to approach too closely the conditions of the pilot experiment. Some experiments were carried out in which this was done, but their results were on the whole less rather than more informative; in one case, the zone of interpenetration drifted so rapidly that one subspecies was eliminated before much of evolutionary significance had happened.

Population size could not be increased to any useful extent because it was limited by the size of direct-access store available and little improvement was possible with the KDF9 machine which was used. It would also have been limited by time. With still faster machines with more direct-access 
store capacity, it will be possible to continue this work with larger populations, and the technique will become increasingly powerful. There are certainly many more problems to investigate in the field of speciation processes and the evolution of genetic discontinuity.

\section{Summary}

1. Computer simulation techniques were used to investigate the population genetics of the zone of contact between interbreeding plant subspecies, in particular to test the hypothesis that where interbreeding leads to hybrids of low fertility (which involves wastage of the gametes concerned in hybridisation), then any genes tending to prevent interbreeding should have a selective advantage and should increase in frequency with a consequent increase in genetic isolation of the subspecies and a decrease in gene flow between them.

2. Two basic experiments were devised, using flowering period as the potential isolating mechanism. In a situation in which hybrids had a fertility of only 25 per cent. of that of the parent subspecies, both of the latter were given equal possibility of genetic variability in date of flowering and length of the flowering period.

3. In a preliminary experiment, the two subspecies were thoroughly intermingled and pollination took place entirely at random through the whole population. At the beginning, the two subspecies flowered simultaneously and there was extensive hybridisation with substantial loss of subspecific identity. But within about 60 generations one subspecies had become lateflowering and the other early-flowering, and both had shortened the length of their flowering periods; there was very little overlap in flowering and consequently there was very little hybridisation; both subspecies had largely regained their identity, and were effectively isolated genetically from one another.

4. In a more complex experiment, the subspecies were in contact but not intermingled, and there was a restriction on the distance of pollen and seed movement. Initially, flowering was not quite simultaneous.

5. In the early stages, hybridisation occurred in the region of contact and there was only slight interpenetration of the two subspecies; hybridisation is a powerful factor in hindering interpenetration. Evolution of floweringtime difference occurred as it had done in the first experiment, but much more slowly. Until such a difference began clearly to appear, the zone of interpenetration of the subspecies (which never lost their identity) remained narrow, but as the difference developed (most strongly in the plants furthest into the territory of the other subspecies) this zone increased in size and eventually there was very considerable intermingling of the subspecies which became virtually sympatric. At the same time there was no increase in the number of hybrids in spite of this much greater intermingling, and this was clearly due to the fact that as a result of selection there had come to be little overlap in flowering period between the subspecies within the zone of interpenetration, and they were now good genetically isolated species.

6. Change in flowering period was not confined to the zone of interpenetration, but the alleles involved flowed outwards into areas well away from it. This was confirmed in an experiment which kept the zone narrow by 
introducing ecological preferences; at the ends of the population, where the subspecies were widely separated from each other and hybridisation was geographically impossible, there came to be a difference of five weeks between their times of maximum flowering.

Acknowledgments.-Most of this work was carried out during a period of sabbatical leave, for which I am very grateful to the Council of the University of Durham. I am also very grateful to Professor E. S. Page for freely putting at my disposal during this period (and at other times) the facilities of the Computing Laboratory of the University of Newcastle-uponTyne, of which he is the Director.

\section{REFERENCES}

CROsBY, J. L. 1961. Teaching genetics with an electronic computer. Heredity, 16, 255-273. CrosBy, J. L. 1963. The evolution and nature of dominance. J. Theoret. Biol., 5, 35-51. CROBBY, J. L. 1966a. Reproductive capacity in the study of evolutionary processes. Reproductive Biology and Taxonomy of Vascular Plants (Ed. J. G. Hawkes for B.S.B.I.), 122-128. Pergamon Press.

CROSBY, J. L. 1966b. Self-incompatibility in the population of Oenothera organensis. Evolution, $20,567-579$.

CRosby, J. L. 1967. Computers in the study of evolution. Sci. Prog. Oxf., 55, 279-292.

Dobzhansky, Th. 1940. Speciation as a stage in evolutionary divergence. Amer. Nat., 74, 312-321.

Emerson, s, 1939. A preliminary survey of the Oenothera organensis population. Genetics, 24, 524-537.

HARPER, J. L. 1966. The reproductive biology of the British poppies. Reproductive Biology and Taxonomy of Vascular Plants (Ed. J. G. Hawkes for B.S.B.I.), 26-39. Pergamon Press.

MAYR, E. 1963. Animal Species and Evolution. Harvard Univ. Press.

MOORE, J. A. 1957. An embryologist's view of the species concept. Amer. Assoc. Adv. Sci. Publ. no. 50 (The Species Problem, Ed. E. Mayr), 325-338.

von Buch, L. 1825. Physicalische Beschreibung der Canarischen Inseln. Kgl. Akad. Wiss., Berlin. (The quoted passage is on pp. 132-133.) 